This is a self-archived - parallel published version of this article in the publication archive of the University of Vaasa. It might differ from the original.

\title{
Multiobjective optimal power flow using a semidefinite programming-based model
}

Author(s): Davoodi, Elnaz; Babaei, Ebrahim; Mohammadi-Ivatloo, Behnam; Shafie-Khah, Miadreza; Catalão, João P. S.

Title: $\quad$ Multiobjective optimal power flow using a semidefinite programmingbased model

Year: $\quad 2020$

Version: Final draft (post print, aam. accepted manuscript)

Copyright C)2020 IEEE. Personal use of this material is permitted. Permission from IEEE must be obtained for all other uses, in any current or future media, including reprinting/republishing this material for advertising or promotional purposes, creating new collective works, for resale or redistribution to servers or lists, or reuse of any copyrighted component of this work in other works.

\section{Please cite the original version:}

Davoodi, E., Babaei, E., Mohammadi-Ivatloo, B., Shafie-Khah, M., \& Catalao, J. P. S., (2020). Multiobjective optimal power flow using a semidefinite programming-based model. IEEE systems journal. https:// doi.org/10.1109/TSTE.2020.2976038 


\title{
Multiobjective Optimal Power Flow Using a Semidefinite Programming-Based Model
}

\author{
Elnaz Davoodi, Ebrahim Babaei ${ }^{\circledR}$, Senior Member, IEEE, Behnam Mohammadi-Ivatloo ${ }^{\circledR}$, Senior Member, IEEE, \\ Miadreza Shafie-Khah, Senior Member, IEEE, and João P. S. Catalão ${ }^{\circledR}$, Senior Member, IEEE
}

\begin{abstract}
In spite of the significant advance achieved in the development of optimal power flow (OPF) programs, most of the solution methods reported in the literature have considerable difficulties in dealing with different-nature objective functions simultaneously. By leveraging recent progress on the semidefinite programming (SDP) relaxations of OPF, in the present article, attention is focused on modeling a new SDP-based multiobjective OPF (MO-OPF) problem. The proposed OPF model incorporates the classical $\varepsilon$-constraint approach through a parameterization strategy to handle the multiple objective functions and produce Pareto front. This article emphasizes the extension of the SDPbased model for MO-OPF problems to generate globally nondominated Pareto optimal solutions with uniform distribution. Numerical results on IEEE 30-, 57-, 118-bus, and Indian utility 62-bus test systems with all security and operating constraints show that the proposed convex model can produce the nondominated solutions with no duality gap in polynomial time, generate efficient Pareto set, and outperform the well-known heuristic methods generally used for the solution of MO-OPF. For instance, in comparison with the obtained results of NSGA-II for the 57-bus test system, the best compromise solution obtained by SDP has $1.55 \%$ and $7.42 \%$ less fuel cost and transmission losses, respectively.
\end{abstract}

Index Terms-Convexification, multiobjective OPF (MO-OPF), optimal power flow (OPF), semidefinite programming (SDP), $\varepsilon$-constraint method.

\section{INTRODUCTION}

$\mathbf{N}$ OWADAYS, humanity's need for electric power energy is increasing day by day, and electrical demands have

Manuscript received September 1, 2019; revised October 27, 2019 and January 6, 2020; accepted January 10, 2020. This work was supported by a Postdoctoral Research Project of the Research Affairs Office of the University of Tabriz, Tabriz, Iran, under Grant S-27-8. (Corresponding author: Ebrahim Babaei; Behnam Mohammadi-Ivatloo.)

E. Davoodi is with the Faculty of Electrical and Computer Engineering, University of Tabriz, Tabriz 51666, Iran (e-mail: e.davoodi@ tabrizu.ac.ir).

E. Babaei is with the Faculty of Electrical and Computer Engineering, University of Tabriz, Tabriz 51666, Iran, and also with the Engineering Faculty, Near East University, Nicosia 99138, North Cyprus, Mersin 10, Turkey (e-mail: e-babaei@tabrizu.ac.ir).

B. Mohammadi-Ivatloo is with the Faculty of Electrical and Computer Engineering, University of Tabriz, Tabriz 51666, Iran, and also with the Institute of Research and Development, Duy Tan University, Da Nang 550000, Vietnam (e-mail: bmohammadi@tabrizu.ac.ir).

M. Shafie-Khah is with the School of Technology and Innovations, University of Vaasa 65200, Vaasa, Finland (e-mail: miadreza@gmail.com).

J. P. S. Catalão is with the Faculty of Engineering, Institute for Systems and Computer Engineering, Technology and Science, University of Porto, 4200-465 Porto, Portugal (e-mail: catalao@fe.up.pt).

Digital Object Identifier 10.1109/JSYST.2020.2971838 increased rapidly concerning the growth in population and industrial applications. Hence, various aspects, such as minimizing fuel costs, losses, environmental pollution, and so on, are essential to be taken into consideration for satisfactory power system operation and planning [1], [2]. For these reasons and more, optimal power flow (OPF) is the heart of an economically efficient operation tool for independent system operators. Given that almost $4 \%$ of the total system costs refer to the power losses so that the economic feasibility of power systems, particularly in a competitive energy market, relies on the optimum set of all parameters related to any generator or transmission element [3]. On the other hand, due to the dependence of transmission losses on reactive power, indeed, optimization of the reactive power dispatch of power systems can be regarded as the minimization of the real power loss.

Hence, the OPF objective functions, which are basically founded on the system economic aspects, require the loss factor besides the cost function to consider, while meeting the various equality and inequality constraints.

In addition, environmental pollution is a matter of concern, which is raised by the harmful effect of the electric power industry. Therefore, the OPF problem is largely classified as a nonlinear constrained multiobjective optimization (MOO) in which the total generation fuel cost, besides the other objective functions, must be inevitably optimized. In a single-objective optimization problem, there exists a global optimum, while in the case of MOO, no optimal solution is evidently defined; instead, a set of solutions, namely the Pareto optimal front, is given [4]. The convex or nonconvex models of the multiobjective programming problem require appropriate approaches to manage the multiobjective nature [5] and are dependent on obtaining an efficient set of Pareto optimal solutions, which is not as simple as it is for a single-objective optimization problem. Therefore, different methods are defined for solving multiobjective optimization problems (MOOPs). One way to handle the multiobjective OPF (MO-OPF) problem is to turn it to a single-objective optimization problem by specific conversion methods, such as weighted sum. With varying the weights of the obtained linear function, all of the points that are on the convex envelope can be found. However, from the literature, some reported drawbacks of the weighted sum method are solutions with nonuniform distribution and not discovering the optimal solutions over the nonconvex areas. The $\varepsilon$-constrained method is also a widely known method to tackle the MOOPs, which generates a noninferior set. In this approach, all but one objective function transformed into constraints, and the Pareto front can be generated exactly by varying the $\varepsilon$-vector. For example, in [6], the MO-OPF problem consisting of simultaneous minimization of the generation cost and transmission losses for the first time is introduced to solve, 
in which a method denominated as a surrogate worth tradeoff is suggested to obtain a best compromised solution (BCS). In another case [7], a MOOP based on the $\varepsilon$-constraint is employed to minimize the operation cost as well as the air pollutant emissions as the second objective function, while the transient stability margin as the third objective function is maximized simultaneously. The $\varepsilon$-constrained method is capable of detecting a number of nondominated solutions on a nonconvex region in contrast to the weighted sum scalarization. Another way to solve the MOOPs is producing all the Pareto optimal solutions or a typical subset of the Pareto frontier. These kinds of methods entitled posterior methods, which are classified into two groups: mathematical programming based posterior approaches, such as normal boundary intersection [8], and evolutionary algorithms. Presently, a number of evolutionary MOO algorithms, including differential evolution (DE) [9], teaching learning-based optimization (TLBO) [10], particle swarm optimization [11], evolutionary programming [12], gravitational search algorithm (GSA) [13], and so on, have been applied to handle MOOPF problems. Evolutionary algorithms can generate sets of the solution and, in general, are able to compute a representative set of the entire Pareto front. A big advantage of evolutionary computation is that it is conceptually not complicated and can be easily applied to different kinds of power system optimization problems [14], but then again, some possible limitations of these algorithms are their computationally burdensome [15] and no guarantee of obtaining the noninferior solutions.

Semidefinite programming (SDP) is a new subfield of convex optimizations, which has fascinated researchers since the discovery of conic sections. SDP can solve optimization problems over positive semidefinite matrices with a linear cost function and linear constraints [16]. A large number of realistic electric power engineering problems consist of polynomial objective functions and constraints, but the applications of SDP in the power system have been reported scantily in the available literature. Recent trends of research in the subfield of OPF generally can be classified into three groups.

1) Investigation of sufficient conditions under which the existence of no duality gap for different OPF problems is guaranteed [17].

2) Employing semidefinite relaxation (SDR) techniques to reduce the computational cost of SDR problems [18].

3) The identification of any limitations of the convexification methods [19].

\section{A. Summary of Contributions}

Due to the inherent nonconvex nature of the multiobjective problems, most of the solution methods focused on finding local solutions and are, thus, only appropriate for convex problems. Another alternative for solving nonconvex problems is using heuristic methods that are very time consuming and inefficient for MOOPs. That is all the more reason to take a special interest in a deterministic framework, which we actually tried to address in this article using the SDP. Recent advances in convex optimization and their application in engineering motivate the authors to use the SDP in the proposed area. Specifically, the main contributions of the proposed framework can be listed pointwise as follows.

1) The proposed article mainly seeks to focus on the further development of the existing OPFs based on the SDP to generalize this model from the other aspects. With this end in view, this article next tries to propose an exact convex model for highly sophisticated nonconvex multiobjective $O P F$ problems with several nonlinear targets and obtain the zero duality gap for more sophisticated problems.

2) In virtue of the nonconvex nature of the multiobjective problem, it is proved that the SDP relaxations converge to the globally optimal solutions of the MO-OPF problem starting from any initial states.

3) The basic idea for producing Pareto front in this article is developing a novel model based on the $\varepsilon$-constraint by establishing a convex scalar dual problem. The resulting convex model advances the application of the $\varepsilon$-constraint method to provide good approximation of the Pareto frontier and avoids inefficient ones.

4) This article initially includes simultaneous minimization of fuel cost and losses in transmission systems and then models total fuel cost, losses, and pollutant emission concurrently as competing for objective functions, which must be moved into the set of constraints and turned into the SDP form.

5) More importantly, the multiobjective model proposed in this article is a general SDP based on the $\varepsilon$-constraint model, which can be developed over the other nonconvex MOOPs with polynomial functions.

The remainder of this article is arranged as follows. Section II describes the basic formulation of the OPF problem. The MOO description is provided in Section III. Section IV gives the singleobjective OPF problems based on the SDP and then introduces the proposed SDR of the MO-OPF problem. This is followed by simulations and comparison with the results with the other well-known state-of-the-art algorithms in Section V. At last, the main conclusion of the article is drawn in Section VI.

\section{Terminology And Problem Statement}

\section{A. Model}

Consider a typical power network, where $\mathcal{N}, \mathcal{N}_{\boldsymbol{b}}$, and $\mathcal{N}_{\mathcal{T}}$ represent, respectively, the set of all buses, the number of shunt VAR compensators, and the set of all tap regulating transformers. $\mathcal{G}$ denotes the set of generator buses, i.e., $\mathcal{G} \subseteq \mathcal{N}$, and $\mathcal{L} \subseteq \mathcal{N} \times \mathcal{N}$ describes the set of all lines such that if there is a transmission line between $k$ and $l$ then $(k, l),(l, k) \in \mathcal{L}$. $T_{i, k} \in \mathcal{N}_{\mathcal{T}}$ shows the transformer available between buses $(i, k) \in \mathcal{L}$. The apparent power of the connected generator and load to the bus $i$ is expressed as $S_{g, i}=P_{g, i}+j Q_{g, i}$ and $S_{d, i}=P_{d, i}+j Q_{d, i}$, respectively. Besides, the apparent power flow from bus $i$ to bus $k$ through $(i, k) \in \mathcal{L} \mathfrak{U}$ is presented as $S_{i, k}$. Stack, respectively, the voltages and currents in common vectors as $\mathbf{v}=\left[v_{1}, v_{2}, \ldots, v_{N}\right]$ and $\mathbf{i}=\left[i_{1}, i_{2}, \ldots, i_{N}\right]$. Note that $N$ signifies the number of all buses. Let us define a component of the nodal admittance matrix $\mathrm{Y} \in \mathcal{R}^{N \times N}$ as $y_{i j}$, where $y_{i j}$ describes the mutual admittance between the buses $i$ and $j$, and $y_{i i}$ shows the total admittance connected to the bus $i$.

\section{B. Objective Functions}

1) Minimization of the Total Fuel Cost $\left(f_{C}\right)$ : The operation $\operatorname{cost} f_{C}$ is the main objective, which should be minimized and defines the fuel cost associated with each generator. This fuel cost curve can be represented as a quadratic convex or a set of piecewise-linear functions. Here, the fuel cost curve is modeled 
as a convex quadratic function [20], [21]

$$
f_{C}\left(\mathbf{P}_{\mathbf{g}}\right)=\sum_{i \in \mathcal{G}} c_{i 2} P_{g, i}^{2}+c_{i 1} P_{g, i}+c_{i 0}
$$

where $c_{i 2}, c_{i 1}$, and $c_{i 0}$ are the fuel cost coefficients and $P_{g, i}$ is the power generation output of the generator $i$.

2) Minimization of Transmission Line Losses $\left(P_{\text {Loss }}\right)$ : The second objective function, which is imposed on the network and must be optimized, is the active power loss $P_{\text {Loss }}$. This function can be defined as follows:

$$
P_{\text {Loss }}=\operatorname{Re}\left(\sum_{i \in \mathcal{N}} v_{i} i_{i}^{*}\right)
$$

3) Minimization of the Pollutant Emission $\left(f_{E}\right)$ : The total emission of generation is related to the fuel consumption of the thermal units and can be considered as the second-order polynomial form, such as the fuel cost function [22]

$$
f_{E}\left(\mathbf{P}_{\mathbf{g}}\right)=\sum_{i \in \mathcal{G}} e_{i 2} P_{g, i}^{2}+e_{i 1} P_{g, i}+e_{i 0}
$$

where $e_{i 2}, e_{i 1}$, and $e_{i 0}$ are defined as the emission coefficients.

4) Constraints: Assuming the $\pi$-equivalent model of the power network, currents, and voltages fulfill the $\mathbf{i}=\mathbf{Y} \mathbf{v}$ equation. In this way, the power balance equation and physical constraints are defined in the following form:

$$
\begin{aligned}
v_{i} i_{i}^{*} & =S_{g, i}-S_{d, i} \quad \forall i \in \mathcal{N} \\
P_{g, i}^{\min } & \leq P_{g, i} \leq P_{g, i}^{\max } \quad \forall i \in \mathcal{G} \\
Q_{g, i}^{\min } & \leq Q_{g, i} \leq Q_{g, i}^{\max } \quad \forall i \in \mathcal{G} \\
v_{i}^{\min } & \leq\left|v_{i}\right| \leq v_{i}^{\max } \quad \forall i \in \mathcal{N} \\
Q_{b, i}^{\min } & \leq Q_{b, i} \leq Q_{b, i}^{\max } \quad \forall i \in \mathcal{N}_{\boldsymbol{b}} \\
T_{i, k}^{\min } & \leq\left|T_{i, k}\right| \leq T_{i, k}^{\max } \quad \forall(i, k) \in \mathcal{L} \\
\left|S_{i, k}\right| & \leq S_{i, k}^{\max } \quad \forall(i, k) \in \mathcal{L} .
\end{aligned}
$$

In the above equation, $\left|v_{i}\right|$ signifies the voltage magnitude of the $i$ th bus. $Q_{b, i}$ denotes the shunt VAR injection of the $i$ th shunt compensator $\left(i \in \mathcal{N}_{\boldsymbol{b}}\right)$. The transformer ratio between bus $i \in \mathcal{N}$ and bus $k \in \mathcal{N}$ belongs to the transformer, $t_{i, k} \in \mathcal{N}_{\mathcal{T}}$ is indicated by $\left|T_{i, k}\right|$, wherein $(i, k) \in \mathcal{L}$. Superscripts "min" and "max" show, respectively, the upper and lower bounds related to the determined variables.

\section{Multiobjective Programming}

\section{A. Model}

Numerous real-world applications entail more than one objective function, which is needed to be optimized concurrently, while a number of associated equality and inequality equations must be satisfied. In this type of optimization, a single solution that optimizes all the objective functions and fulfills the constraints does not exist. Rather, in that case, researchers deal with a set of nondominated solutions. The following problem defines the general formulation of multiobjective programming problems:

$$
\begin{aligned}
& \operatorname{minimize} \mathbf{f}(\mathbf{x}, \mathbf{u}) \\
& \quad \text { subject to } \mathbf{h}(\mathbf{x}, \mathbf{u})=0, \quad \mathbf{g}(\mathbf{x}, \mathbf{u}) \leq 0
\end{aligned}
$$

Here, $\mathbf{x}$ is the vector of state variables containing slack bus power, load bus voltages, reactive power generator outputs, and transmission line loading. $\mathbf{u}$ is the vector of control variables comprising of generator real power outputs except for the slack bus, generator voltages, transformer tap settings, and reactive power injections. $\mathbf{f}(\mathbf{x}, \mathbf{u})$ is the set of objective functions as $\mathbf{f}(\mathbf{x}, \mathbf{u})=\left[f_{1}(\mathbf{x}, \mathbf{u}), f_{2}(\mathbf{x}, \mathbf{u}), \ldots, f_{n_{f}}(\mathbf{x}, \mathbf{u})\right]^{T}$. Besides, $\mathbf{h}(\mathbf{x}, \mathbf{u})$ and $\mathbf{g}(\mathbf{x}, \mathbf{u})$ denote, respectively, the equality and inequality constraints' vectors, i.e., illustrating the power balance as well as the upper and lower limits of variables. The number of objective functions is defined by $n_{f}$.

\section{B. Semidefinite Programming}

SDP can be defined as a generalization of linear programming over the intersection of the cone of positive semidefinite matrices with an affine space and let us define a semidefinite constraint, in addition to the linear constraints set. SDP has an important role in the combinatorial optimizations, where it is utilized to deal with the nonlinear convex relaxations of quadratic NP-hard optimization problems [23]. Then, all linear programs can be converted as SDPs, and the polynomial optimization problems can also be reformulated as an SDP form. Recall that the semidefiniteness of a matrix is indicated by " $\succeq$," i.e., IF $A \succeq B$ THEN $A-B$ is positive semidefinite $(A-B \succeq 0)$, and the eigenvalues of the matrix $A-B$ are all non-negative.

Using convex approaches for solving the OPF problem may lead to some challenges arisen from NP-hardness and nonconvexity of a set of limitations. This matter can be handled by converting a feasible set of the problem into a convex one. In other words, constraints and objective functions in the nonlinear form are changed to linear ones by replacing them into the SDP variables and constraints form. A salient feature of SDP is that the dual of SDP is also in the SDP form and the associated dual problem can be obtained by applying Shor's relaxation. Therefore, if the obtained duality gap is zero, then strong duality is held, and the Dual Lagrangian problem can find the best possible bound. Although strong duality does not easily obtain, some conditions are generally needed to place on the problem under which a strong duality is obtained. One of these so-called "constraint qualifications" is Slater's condition. This means, if the primal problem is convex and Slater's condition is satisfied, then strong duality is held, and the primal problem along with the dual problem can have the same solutions [24].

\section{C. $\varepsilon$-Constraint Scalarization Method}

As previously noted, the decision makers attempt to find the "most preferred" alternative among the obtained solutions through solving a multiobjective problem. To do so, the primal multiobjective problem should be turned into a single-objective one, and a collection of noninferior alternatives should be obtained. This is regarded as a scalarized problem used for changing a multiobjective problem into a simple single-objective problem. It should be noted that the appropriate solutions give a guarantee for obtaining the optimal Pareto set [25]. A brief review of the $\varepsilon$-constraint method can be found in [26].

1) $\varepsilon$-Constraint: The main objective function is singled out in the $\varepsilon$-constraint method, and the other ones are considered as 
inequality constraints, as follows:

$$
\begin{aligned}
& \min \quad f_{m}(\mathbf{x}, \mathbf{u}) \\
& \text { subject to } \mathbf{h}(\mathbf{x}, \mathbf{u})=0 \\
& \quad \mathbf{g}(\mathbf{x}, \mathbf{u}) \leq 0, \quad f_{i}(\mathbf{x}, \mathbf{u}) \leq \varepsilon_{i}, \quad i=1, \ldots, n_{f} \text { and } i \neq m
\end{aligned}
$$

where $f_{i}(\mathbf{x}, \mathbf{u})$ defines the objective functions switched over to the inequality constraints and $\varepsilon_{i}$ is the upper bound of the $i$ th extra inequality constraint and $\mathrm{x}$ represents the vector of decision variables, which comprises dependent and control variables.

\section{PROPOSED MODEL}

\section{A. Reformulation and SDP Relaxation}

Generally, the presence of the multiple local minima of a problem makes it a big challenge in global optimization. Intrinsically, the nonlinear interrelation among the physical parameters of the electrical network causes nonlinearity and nonconvexity of the OPF problem [27]. On the other hand, taking account of several objectives created the additional complexity of the problem. Hence, convexifying this fundamental problem can make the problem be solved more efficiently than a general case. It should be noticed that a MOOP is convex if all objective functions and the feasible region are convex. This section represents the reformulation of the MO-OPF problem with economic, loss, and emission minimization objectives into a framework that permits it to be handled as an SDP form. To do so, let $e_{i}$ denote the $i$ th column of the standard basis and $v_{i}=v_{d i}+j v_{q i}$ denote the complex voltage of bus $i \in \mathcal{N}$. Consider the vector $\mathbf{V} \in \mathcal{R}^{2 N}$ comprising the real and imaginary parts of the voltage coordinates as $\mathbf{V}=\left[v_{d 1}, \ldots, v_{d N}, v_{q 1}, \ldots, v_{q N}\right]$. Using the admittance matrix $\mathbf{Y}$, some new matrices are defined as $Y_{i}:=e_{i} e_{i}^{T} \mathbf{Y}$ and $\mathrm{Y}_{i, k}=\left(\tilde{y}_{i, k}+y_{i, k}\right) e_{i} e_{i}^{T}-\left(y_{i, k}\right) e_{k} e_{k}^{T}$, and then based on these matrices, some other necessary constant matrices, namely $\mathbf{Y}_{i}, \tilde{\mathbf{Y}}_{i}, \mathbf{Y}_{i, k}, \tilde{\mathbf{Y}}_{i, k}$, can be described as follows:

$$
\begin{aligned}
\mathbf{Y}_{i} & :=\frac{1}{2}\left(Y_{i}+Y_{i}^{\mathcal{H}}\right) \quad \tilde{\mathbf{Y}}_{i}:=\frac{j}{2}\left(Y_{i}-Y_{i}^{\mathcal{H}}\right) \\
\mathbf{Y}_{i, k} & :=\frac{1}{2}\left(Y_{i, k}+Y_{i, k}^{\mathcal{H}}\right) \quad \tilde{\mathbf{Y}}_{i, k}:=\frac{j}{2}\left(Y_{i, k}-Y_{i, k}^{\mathcal{H}}\right) \\
\mathbf{M}_{i} & :=e_{i} e_{i}^{T}
\end{aligned}
$$

where the superscript $\mathcal{H}$ points out the conjugate transpose of the related matrix. The above matrices are described in more detail in the Appendix.

Using the above matrices, the OPF parameters can be rewritten. In view of these matrices, squared voltage magnitude is represented as $\operatorname{tr}\left(\mathbf{V}^{T} \mathbf{M}_{i} \mathbf{V}\right)$, wherein "tr" demonstrates the trace of a square matrix. Recall that the sum of diagonal components is known as the trace of a matrix. The net active and reactive power injections at the bus $i$ are defined as $P_{\text {inj }, i}=P_{g, i}-P_{d, i}$ and $Q_{\text {inj }, i}=Q_{g, i}-Q_{d, i} \forall i \in \mathcal{G}$, which can be rewritten by the above matrices as $P_{\mathrm{inj}, i}=\operatorname{tr}\left(\mathbf{V}^{T} \mathbf{Y}_{i} \mathbf{V}\right)$ and $Q_{\text {inj, } i}=\operatorname{tr}\left(\mathbf{V}^{T} \tilde{\mathbf{Y}}_{i} \mathbf{V}\right) \forall i \in \mathcal{N}$. Besides, the active and reactive power transferred from bus $i \in \mathcal{N}$ to bus $k \in \mathcal{N}$ are described, respectively, as $P_{i, k}=\operatorname{tr}\left(\mathbf{V}^{T} \mathbf{Y}_{i, k} \mathbf{V}\right)$ and $Q_{i, k}=$ $\operatorname{tr}\left(\mathbf{V}^{T} \tilde{\mathbf{Y}}_{i, k} \mathbf{V}\right)$. Assume that each bus $i \in \mathcal{N}$ includes a variable shunt element within a specified range. The upper and lower bounds of these elements are fixed at zero if associated buses have no shunts. Accordingly, (4c) can be rewritten as follows:

$$
\begin{aligned}
& Q_{g, i}^{\min }-Q_{d, i}+Q_{b, i} \leq Q_{\mathrm{inj}, i} \leq Q_{g, i}^{\max }-Q_{d, i}+Q_{b, i} \quad \forall i \in \mathcal{N} \\
& \quad\left(\text { if } i \in \mathcal{N} \backslash \mathcal{G} \text { then } Q_{g, i}^{\max }\right. \\
& \left.\quad=Q_{g, i}^{\min }=0, \quad \text { if } i \notin \mathcal{N}_{b} \text { then } Q_{b, i}=0\right) .
\end{aligned}
$$

Besides, tap ratios of the transformers are considered as the other controllable parameters. Taking unknown tap ratios of the two-port, $\Pi$ block of the transformer into consideration, are appeared in a nonlinear form in the admittance matrix. To avoid this issue, another type of modeling must be employed [28]. To this end, assume that every tap-changing transformer connected between buses $i$ and $k$ in the system is replaced with an ideal transformer connected between two real/virtual buses $i$ and $k^{\prime}$, along with some lumped elements (series resistance, leakage reactance, and so on). Define $P_{i, k^{\prime}}$ and $Q_{i, k^{\prime}}$ as the active and reactive powers transferred from the bus $i$ to the rest of the network and consider the admittance matrix $Y$ after eliminating the transformer. Consider the line flow limits for all lines, apart from the lines, including transformers. Furthermore, add the extra condition related to the lines with transformers as $P_{i, k}^{\prime 2}+Q_{i, k}^{\prime 2} \leq\left(S_{i, k^{\prime}}^{\max }\right)^{2}$. Actually, modeling a transformer in this way may be needed to define a virtual bus such as $k^{\prime}$. Note that the transformer is considered ideal, and the admittance shown is related to the line $(i, k) \in \mathcal{L}$ and may be for the nonideal transformer (before modeling). Additional information about this modeling is available in [28].

\section{B. MO-OPF Problem Formulated as SDP}

1) Modeling of the MO-OPF Problem Considering two Objective Functions: Generally, the MO-OPF problem, with the goal of minimization of production cost and transmission losses, can be arranged as an aggregation of the selected objective functions and constraints as follows:

$$
\begin{aligned}
& \min \left[f_{C}(\mathbf{x}, \mathbf{u}), P_{\text {Loss }}(\mathbf{x}, \mathbf{u})\right] \\
& \text { subject to }(4 \mathrm{a})-(4 \mathrm{~g})
\end{aligned}
$$

where $f_{C}$ and $P_{\text {Loss }}$ describe the cost and loss functions, respectively. Accordingly, equivalent reformulation of the MO-OPF problem formula, introducing a new variable $W:=\mathbf{V V}^{T}$ can be expressed as problem (12). It should be noticed that a given matrix $W$ is equivalent to $\mathbf{V} \mathbf{V}^{T}$ if and only if $W$ is a rank-one symmetric positive semidefinite matrix

$$
\begin{aligned}
\min & {\left[\sum _ { i \in \mathcal { G } } \left\{c_{i 2}\left(\operatorname{tr}\left(\mathbf{Y}_{i} W\right)+P_{d, i}\right)^{2}\right.\right.} \\
& \left.\left.+c_{i 1}\left(\operatorname{tr}\left(\mathbf{Y}_{i} W\right)+P_{d, i}\right)+c_{i 0}\right\}, \sum_{i \in \mathcal{N}} \operatorname{tr}\left(\mathbf{Y}_{i} W\right)\right]
\end{aligned}
$$

subject to

$P_{g, i}^{\min }-P_{d, i} \leq \operatorname{tr}\left(\mathbf{Y}_{i} \boldsymbol{W}\right)+P_{i, k^{\prime}} \quad \forall i, k^{\prime} \in \mathcal{N}\left(\right.$ if $i, k^{\prime} \in \mathcal{N} \backslash \mathcal{G}$
then $P_{g, i}^{\min }=0, \quad$ if $\left(i, k^{\prime}\right) \notin \mathcal{N}_{\mathcal{T}}$ then $\left.P_{i, k^{\prime}}=0\right) \quad(12 \mathrm{~b})$
$\operatorname{tr}\left(\mathbf{Y}_{i} \boldsymbol{W}\right)+P_{i, k^{\prime}} \leq P_{g, i}^{\max }-P_{d, i} \quad \forall i, k^{\prime} \in \mathcal{N}\left(\right.$ if $i, k^{\prime} \in \mathcal{N} \backslash \mathcal{G}$
then $P_{g, i}^{\max }=0, \quad$ if $\left(i, k^{\prime}\right) \notin \mathcal{N}_{\mathcal{T}}$ then $\left.P_{i, k^{\prime}}=0\right)$ 


$$
\begin{aligned}
& Q_{g, i}^{\min }-Q_{d, i}+Q_{b, i} \leq \operatorname{tr}\left(\tilde{\mathbf{Y}}_{i} W\right) \\
& \quad+Q_{i, k^{\prime}} \quad \forall i, k^{\prime} \in \mathcal{N}\left(\text { if } i, k^{\prime} \in \mathcal{N} \backslash \mathcal{G}\right.
\end{aligned}
$$

then $Q_{g, i}^{\min }=0$, if $i \notin \mathcal{N}_{b}$ then

$$
\begin{aligned}
& \left.Q_{b, i}=0, \quad \text { if }\left(i, k^{\prime}\right) \notin \mathcal{N}_{\mathcal{T}} \text { then } Q_{i, k^{\prime}}=0\right) \\
& \operatorname{tr}\left(\tilde{\mathbf{Y}}_{i} \boldsymbol{W}\right)+Q_{i, k^{\prime}} \leq Q_{i}^{\max }-Q_{d, i} \\
& \quad+Q_{b, i} \quad \forall i, k^{\prime} \in \mathcal{N}\left(\text { if } i, k^{\prime} \in \mathcal{N} \backslash \mathcal{G}\right.
\end{aligned}
$$

then $Q_{i}^{\max }=0$, if $i \notin \mathcal{N}_{b}$ then

$$
\begin{aligned}
& \left.Q_{b, i}=0, \quad \text { if }\left(i, k^{\prime}\right) \notin \mathcal{N}_{\mathcal{T}} \text { then } Q_{i, k^{\prime}}=0\right) \\
& \left(v_{i}^{\min }\right)^{2} \leq \operatorname{tr}\left(\mathbf{M}_{i} \boldsymbol{W}\right) \quad \forall i \in \mathcal{N} \\
& \operatorname{tr}\left(\mathbf{M}_{i} \boldsymbol{W}\right) \leq\left(v_{i}^{\max }\right)^{2} \quad \forall i \in \mathcal{N} \\
& Q_{b, i}^{\min } \leq Q_{b, i} \quad \forall i \in \mathcal{N}_{b} \\
& Q_{b, i} \leq Q_{b, i}^{\max } \quad \forall i \in \mathcal{N}_{b} .
\end{aligned}
$$

Equations (12j)-(12o) are corresponding to the transformers [28]

$$
\begin{aligned}
& \left(T_{i, k^{\prime}}^{\min }\right)^{2} \operatorname{tr}\left(\mathbf{M}_{k^{\prime}} \boldsymbol{W}\right) \leq \operatorname{tr}\left(\mathbf{M}_{i} \boldsymbol{W}\right) \quad \forall\left(i, k^{\prime}\right) \in \mathcal{N}_{T} \\
& \operatorname{tr}\left(\mathbf{M}_{i} \boldsymbol{W}\right) \leq\left(T_{i, k^{\prime}}^{\max }\right)^{2} \operatorname{tr}\left(\mathbf{M}_{i} \boldsymbol{W}\right) \quad \forall\left(i, k^{\prime}\right) \in \mathcal{N}_{T} \\
& \boldsymbol{W}_{i, N+k^{\prime}}=\boldsymbol{W}_{k^{\prime}, N+i} \\
& \boldsymbol{W}_{i, k^{\prime}} \succeq 0 \\
& \boldsymbol{W}_{N+i, N+k^{\prime}} \succeq 0 \\
& {\left[\begin{array}{ccc}
-\left(S_{i, k^{\prime}}^{\max }\right)^{2} & P_{i, k^{\prime}} & Q_{i, k^{\prime}} \\
P_{i, k^{\prime}} & -1 & 0 \\
Q_{i, k^{\prime}} & 0 & -1
\end{array}\right] \preceq 0 \quad \forall\left(i, k^{\prime}\right) \in \mathcal{N}_{\mathcal{T}}} \\
& {\left[\begin{array}{ccc}
-\left(S_{i, k}^{\max }\right)^{2} & \operatorname{tr}\left(\mathbf{Y}_{i, k} \boldsymbol{W}\right) & \operatorname{tr}\left(\tilde{\mathbf{Y}}_{i, k} \boldsymbol{W}\right) \\
\operatorname{tr}\left(\mathbf{Y}_{i, k} \boldsymbol{W}\right) & -1 & 0 \\
\operatorname{tr}\left(\tilde{\mathbf{Y}}_{i, k} \boldsymbol{W}\right) & 0 & -1
\end{array}\right]} \\
& \preceq 0 \quad \forall(i, k) \in \mathcal{L} \operatorname{and}(i, k) \notin \mathcal{N}_{\mathcal{T}} \\
& \boldsymbol{W} \succeq 0 \\
& \operatorname{rank}(\boldsymbol{W})=1 .
\end{aligned}
$$

Note that Schur's complement formula is used to conclude that (12o) and (12p) are equivalent to the line flow constraints corresponding to the lines with and without transformers, respectively. The constraint (12l) can be rewritten as

$$
\operatorname{tr}\left(\mathbf{M}_{i, N+k^{\prime}}^{\prime} W\right)=\operatorname{tr}\left(\mathbf{M}_{k^{\prime}, N+i}^{\prime} \boldsymbol{W}\right) \quad \forall\left(i, k^{\prime}\right) \in \mathcal{N}_{\mathcal{T}} .
$$

Besides, the constraints $(12 \mathrm{~m})$ and $(12 \mathrm{n})$ are rewritten as follows:

$$
\begin{aligned}
\operatorname{tr}\left(\mathbf{M}^{\prime}{ }_{i, k^{\prime}} W\right) \geq 0 \quad \forall\left(i, k^{\prime}\right) \in \mathcal{N}_{\mathcal{T}} \\
\operatorname{tr}\left(\mathbf{M}^{\prime}{ }_{N+i, N+k^{\prime}} W\right) \geq 0 \quad \forall\left(i, k^{\prime}\right) \in \mathcal{N}_{\mathcal{T}}
\end{aligned}
$$

where $\mathbf{M}_{m, n}^{\prime}$ shows a $2 N \times 2 N$ matrix whose $(m, n)$ and $(n, m)$ entries are equal to 1 and 0 otherwise. In the whole modeling process, first, it is presumed that all buses are equipped with generators and shunt elements, and if some bus $i$ does not have one of these parameters, the associated extreme values are set to zero. Besides, whenever the bus $i$ is not connected to any load, then $P_{d, i}+j Q_{d, i}=0$.

2) Modeling of the MO-OPF Problem Considering Three Objective Functions: Primal problem considering three objective functions, i.e., minimization of production cost, transmission losses, and pollutant emission can be defined as (16),

$$
\begin{aligned}
& \min \left[f_{C}(\mathbf{x}, \mathbf{u}), P_{\text {Loss }}(\mathbf{x}, \mathbf{u}), f_{E}(\mathbf{x}, \mathbf{u})\right] \\
& \text { subject to }(4 \mathrm{a})-(4 \mathrm{~g}) .
\end{aligned}
$$

To model the MO-OPF problem with three objective functions, based on the SDP formulation, the following general scheme is proposed:

$$
\begin{gathered}
\min \left[\sum _ { i \in \mathcal { G } } \left\{c_{i 2}\left(\operatorname{tr}\left(\mathbf{Y}_{i} \boldsymbol{W}\right)+P_{d, i}\right)^{2}+c_{i 1}\left(\operatorname{tr}\left(\mathbf{Y}_{i} \boldsymbol{W}\right)+P_{d, i}\right)\right.\right. \\
\left.+c_{i 0}\right\}, \sum_{i \in \mathcal{N}} \operatorname{tr}\left(\mathbf{Y}_{i} W\right), \sum_{i \in \mathcal{G}} e_{i 2} \\
\left.\times\left\{\operatorname{tr}\left(\mathbf{Y}_{i} \boldsymbol{W}\right)+P_{d, i}\right\}^{2}+e_{i 1}\left\{\operatorname{tr}\left(\mathbf{Y}_{i} \boldsymbol{W}\right)+P_{d, i}\right\}+e_{i 0}\right]
\end{gathered}
$$

$$
\text { subject to }(12 \mathrm{~b})-(12 \mathrm{k}),(12 \mathrm{o})-(12 \mathrm{r}),(13)-(15) \text {. }
$$

\section{SDP Relaxation of the Multiobjective Problems}

Actually, at this point, it seems that problems (12) and (17) are as much difficult as problems (11) and (16), respectively, and there is no significant change. However, the reformulated version reveals us to detect the major difficulty in handling problems (12) and (17). Indeed, the rank-one constraint $\operatorname{rank}(W)=1$ is the only nonconvex constraint in these problems. Accordingly, we can drop it to attain the following SDR framework of the OPF problem (12):

$$
\begin{aligned}
\min \left[\sum _ { i \in \mathcal { G } } \left\{c_{i 2}\left(\operatorname{tr}\left(\mathbf{Y}_{i} \boldsymbol{W}\right)+P_{d, i}\right)^{2}\right.\right. \\
\left.\left.\quad+c_{i 1}\left(\operatorname{tr}\left(\mathbf{Y}_{i} \boldsymbol{W}\right)+P_{d, i}\right)+c_{i 0}\right\}, \sum_{i \in \mathcal{N}} \operatorname{tr}\left(\mathbf{Y}_{i} \boldsymbol{W}\right)\right]
\end{aligned}
$$

subject to $(12 \mathrm{~b})-(12 \mathrm{k}),(12 \mathrm{o})-(12 \mathrm{q}),(13)-(15)$.

The general SDR framework for the triobjective problem can be formulated as follows:

$$
\begin{aligned}
& \min \left[\sum _ { i \in \mathcal { G } } \left\{c_{i 2}\left(\operatorname{tr}\left(\mathbf{Y}_{i} \boldsymbol{W}\right)+P_{d, i}\right)^{2}\right.\right. \\
&\left.+c_{i 1}\left(\operatorname{tr}\left(\mathbf{Y}_{i} \boldsymbol{W}\right)+P_{d, i}\right)+c_{i 0}\right\}, \sum_{i \in \mathcal{N}} \operatorname{tr}\left(\mathbf{Y}_{i} W\right), \\
&\left.\sum_{i \in \mathcal{G}} e_{i 2}\left\{\operatorname{tr}\left(\mathbf{Y}_{i} \boldsymbol{W}\right)+P_{d, i}\right\}^{2}+e_{i 1}\left\{\operatorname{tr}\left(\mathbf{Y}_{i} \boldsymbol{W}\right)+P_{d, i}\right\}+e_{i 0}\right]
\end{aligned}
$$

subject to $(18 b)$. 
In order to generate Pareto optimal solutions, the $\varepsilon$-constraint method assumes that the objectives can be arranged according to their importance. Accordingly, let us assume that the cost function is the most important and the minimization of transmission losses, and pollutant emissions are in the next ranks. Therefore, a sequence of single-objective optimization problems based on the SDP formulation for two and more objective functions must be solved as (20) and (21), respectively, such that each run generates one Pareto efficient solution

$$
\begin{aligned}
\min \sum_{i \in \mathcal{G}}\left\{c_{i 2}\left(\operatorname{tr}\left(\mathbf{Y}_{i} \boldsymbol{W}\right)+P_{d, i}\right)^{2}\right. \\
\left.+c_{i 1}\left(\operatorname{tr}\left(\mathbf{Y}_{i} \boldsymbol{W}\right)+P_{d, i}\right)+c_{i 0}\right\}
\end{aligned}
$$

subject to $(18 b)$

$$
\sum_{i \in \mathcal{N}} \operatorname{tr}\left(\mathbf{Y}_{i} \boldsymbol{W}\right) \leq \varepsilon_{2, n_{2}}, \quad n_{2}=0,1, \ldots, p_{2}
$$

and

$$
\begin{aligned}
\min \sum_{i \in \mathcal{G}}\{ & c_{i 2}\left(\operatorname{tr}\left(\mathbf{Y}_{i} \boldsymbol{W}\right)+P_{d, i}\right)^{2} \\
& \left.+c_{i 1}\left(\operatorname{tr}\left(\mathbf{Y}_{i} \boldsymbol{W}\right)+P_{d, i}\right)+c_{i 0}\right\}
\end{aligned}
$$

subject to $(18 \mathrm{~b})$

$$
\begin{aligned}
& \sum_{i \in \mathcal{N}} \operatorname{tr}\left(\mathbf{Y}_{i} \boldsymbol{W}\right) \leq \varepsilon_{2, n_{2}}, \quad n_{2}=0,1, \ldots, p_{2} \\
& \sum_{i \in \mathcal{G}} e_{i 2}\left\{\operatorname{tr}\left(\mathbf{Y}_{i} W\right)+P_{d, i}\right\}^{2}+e_{i 1}\left\{\operatorname{tr}\left(\mathbf{Y}_{i} W\right)+P_{d, i}\right\} \\
& \quad+e_{i 0} \leq \varepsilon_{3, n_{3}}, \\
& n_{3}=0,1, \ldots, p_{3} .
\end{aligned}
$$

Here, $p_{2}$ and $p_{3}$ represent the density of Pareto solutions related to the minimization of loss and pollutant emission, respectively. Recall that the different values of parameters located within the right sides of limited objective functions lead to different results, which can be obtained according to the range of these functions.

The OPF problem is, in general, a highly nonconvex and NP-hard problem. Actually, extracting the optimal solution of the problem $\mathbf{V}^{*}$, which is rank one, from a globally optimum $\boldsymbol{W}^{*}$, is deep trouble. Hence, handling the dual problem of the reformulated relaxed problem may help. To do so, first of all, the Lagrange multiplier of each semidefinite constraint must be defined. $\underline{\lambda}_{i}, \gamma_{i}, \mu_{i}, \beta_{i}$, and $\underline{\tau}_{i}$ are used to represent the Lagrange multipliers corresponding to lower inequality constraints related to the active power (12b), reactive power (12d), the magnitude of voltage (12f), injected reactive power by shunt element (12h), and transformer tap configuration (12j), respectively. Moreover, the Lagrange multipliers connected to upper inequality limitations related to active power $(12 \mathrm{c})$, reactive power $(12 \mathrm{e})$, voltage magnitude $(12 \mathrm{~g})$, injected reactive power by shunt element (12i), and transformer tap configuration (12k) are considered, respectively, by $\bar{\lambda}_{i}, \bar{\gamma}_{i}, \bar{\mu}_{i}, \bar{\beta}_{i}$, and $\bar{\tau}_{i}$, respectively. Lagrange multipliers for constraints (13)-(15) are denoted by $\chi_{i, k}^{\prime}, \chi_{i, k}^{\prime \prime}$, and $\chi_{i, k}^{\prime \prime \prime}$, respectively. In addition, the Lagrange multipliers related to positive semidefinite matrices in (12o) and (12p) are considered $y$ as two $3 \times 3$ symmetric matrices with $\ell_{i, k}^{m n}$ entries in which for every line $(i, k) \in \mathcal{L}$ maybe $(i, k) \in / \notin \mathcal{N}_{\mathcal{T}}$ and for $m \neq n \rightarrow \ell_{i, k}^{m n}=\ell_{i, k}^{n m}$. Note that the quadratic production cost function can be stated by the epigraph formulation $f_{C}\left(\mathbf{P}_{\mathbf{g}}\right) \leq \sum_{i \in \mathcal{G}} t_{f_{C}, i}, \quad t_{f_{C}, i} \geq 0$ and then by implementing the concept of Schur's complement, can be reformulated as

$$
\begin{aligned}
& {\left[\begin{array}{cc}
c_{i 1} \operatorname{tr}\left(\mathbf{Y}_{i} \boldsymbol{W}\right)-t_{f_{C}, i}+a_{f_{c}, i} & \sqrt{c_{i 2}} \operatorname{tr}\left(\mathbf{Y}_{i} \boldsymbol{W}\right)+b_{f_{C}, i} \\
\sqrt{c_{i 2}} \operatorname{tr}\left(\mathbf{Y}_{i} \boldsymbol{W}\right)+b_{f_{C}, i} & -1
\end{array}\right]} \\
& \quad \preceq 0 \quad \forall i \in \mathcal{G}
\end{aligned}
$$

where the unknown parameters used in (22) are described as

$$
\left\{\begin{array}{l}
a_{f_{C}, i}:=c_{i 0}+c_{i 1} P_{d, i} \\
b_{f_{C}, i}:=\sqrt{c_{i 2}} P_{d, i}
\end{array}\right.
$$

and $t_{f_{C}, i}$ is some scalar variable. Therefore, a $2 \times 2$ symmetric matrix as

$$
R_{f_{C}, i}:=\left[\begin{array}{cc}
1 & R_{f_{C}, i}^{12} \\
R_{f_{C}, i}^{12} & R_{f_{C}, i}^{22}
\end{array}\right]
$$

is needed to demonstrate the Lagrange multipliers associated with the positive semidefinite matrix of the cost function. Besides, the Lagrange multiplier related to the inequality constraint (21c) is indicated by $\eta_{\varepsilon_{2}, n_{2}}$. Finally, consider the set of dual multipliers related to (12b)-(12k), (14), (15), and (20c) constraints by $Z$, the linear matrix inequalities subject to (12o)-(12q) and (22) by $R$ and the linear equality constraint (13) by $R^{\prime}$. Now, the dual OPF problem based on the SDP formulation can be modeled using defined Lagrange multipliers.

\section{Dual $O P F$}

1) Dual OPF: Dual problem of single-objective OPF problem considering the minimization of fuel cost as an objective function.

Define a real-valued function $\varphi$

$$
\begin{aligned}
\varphi\left(Z, R, R^{\prime}\right):= & \sum_{i \in \mathcal{N}}\left\{\underline{\lambda}_{i} P_{g, i}^{\min }-\bar{\lambda}_{i} P_{g, i}^{\max }+\lambda_{f_{C}, i} P_{d, i}+\underline{\gamma}_{i} Q_{g, i}^{\min }\right. \\
& -\bar{\gamma}_{i} Q_{g, i}^{\max }+\gamma_{i} Q_{d, i}+\underline{\mu}_{i}\left(v_{\min }\right)^{2}-\bar{\mu}_{i}\left(v_{\max }\right)^{2} \\
& \left.+\underline{\beta}_{i} Q_{b, i}^{\min }-\bar{\beta}_{i} Q_{b, i}^{\max }\right\}+\sum_{i \in \mathcal{G}}\left\{c_{i 0}-R_{f_{C}, i}^{22}\right\} \\
& -\sum_{(i, k) \in \mathcal{L} \& \notin \mathcal{N}_{\mathcal{T}}}\left\{\left(S_{i, k}^{\max }\right)^{2} \ell_{i, k}^{11}+\ell_{i, k}^{22}+\ell_{i, k}^{33}\right\} \\
& -\sum_{\left(i, k^{\prime}\right) \in \mathcal{N}_{\mathcal{T}}}\left\{\left(S_{i, k^{\prime}}^{\max }\right)^{2} \ell_{i, k^{\prime}}^{11}+\ell_{i, k^{\prime}}^{22}+\ell_{i, k^{\prime}}^{33}\right\}
\end{aligned}
$$

and define linear matrix inequality $\mathbf{A}$

$$
\begin{aligned}
& \mathbf{A}\left(Z, R, R^{\prime}\right):=\sum_{i \in \mathcal{N}}\left\{\lambda_{f_{C}, i} \mathbf{Y}_{i}+\gamma_{i} \tilde{\mathbf{Y}}_{i}+\mu_{i} M_{i}\right\} \\
& +\sum_{(i, k) \in \mathcal{L}, \notin \mathcal{N}_{\mathcal{T}}}\left\{2 \ell_{i, k}^{12} \mathbf{Y}_{i, k}+2 \ell_{i, k}^{13} \tilde{\mathbf{Y}}_{i, k}\right\} \\
& +\sum_{\left(i, k^{\prime}\right) \in \mathcal{N}_{T}}\left\{\mathbf{M}_{k^{\prime}}\left(\underline{\tau}_{i, k^{\prime}}\left(T_{i, k^{\prime}}^{\min }\right)^{2}-\bar{\tau}_{i, k^{\prime}}\left(T_{i, k^{\prime}}^{\max }\right)^{2}\right)+\tau_{i, k^{\prime}} \mathbf{M}_{i}\right.
\end{aligned}
$$




$$
\begin{aligned}
& +\chi_{i, k^{\prime}}^{\prime}\left(\mathbf{M}_{i, N+k^{\prime}}^{\prime}-\mathbf{M}_{k^{\prime}, N+i}^{\prime}\right)-\chi_{i, k^{\prime}}^{\prime \prime} \mathbf{M}_{i, k^{\prime}}^{\prime} \\
& \left.-\chi^{\prime \prime \prime}{ }_{i, k^{\prime}} \mathbf{M}^{\prime}{ }_{N+i, N+k^{\prime}}\right\}
\end{aligned}
$$

where

$$
\lambda_{f_{C}, i}:= \begin{cases}-\underline{\lambda}_{i}+\bar{\lambda}_{i}+c_{i 1}+2 R_{f_{C}, i}^{12} \sqrt{c_{i 2}} & \forall i \in \mathcal{G} \\ -\underline{\lambda}_{i}+\bar{\lambda}_{i}, & \text { otherwise }\end{cases}
$$

and

$$
\begin{cases}\gamma_{i}:=-\underline{\gamma}_{i}+\bar{\gamma}_{i} & \forall i \in \mathcal{N}, \tau_{i, k^{\prime}}:=-\underline{\tau}_{i, k^{\prime}}+\bar{\tau}_{i, k^{\prime}} \\ \mu_{i}:=-\underline{\mu}_{i}+\bar{\mu}_{i} & \forall\left(i, k^{\prime}\right) \in \mathcal{N}_{\mathcal{T}}\end{cases}
$$

Then, the dual OPF problem taking minimization of total fuel cost into account as the objective function is structured as

$$
\max \varphi\left(Z, R, R^{\prime}\right)
$$

subject to

$$
\begin{aligned}
& \mathbf{A}\left(Z, R, R^{\prime}\right) \succeq 0, \quad R \succeq 0, \quad Z \geq 0 \\
& \underline{\gamma}_{i}-\bar{\gamma}_{i}-\underline{\beta}_{i}+\bar{\beta}_{i}=0 \quad \forall i \in \mathcal{N}_{b} \\
& -\underline{\lambda}_{i}+\bar{\lambda}_{i}+\underline{\lambda}_{k^{\prime}}-\bar{\lambda}_{k^{\prime}}+2 \ell_{i, k^{\prime}}^{12}=0 \quad \forall\left(i, k^{\prime}\right) \in \mathcal{N}_{\mathcal{T}} \\
& -\underline{\gamma}_{i}+\bar{\gamma}_{i}+\underline{\gamma}_{k^{\prime}}-\bar{\gamma}_{k^{\prime}}+2 \ell_{i, k^{\prime}}^{13}=0 \quad \forall\left(i, k^{\prime}\right) \in \mathcal{N}_{\mathcal{T}} .
\end{aligned}
$$

Dual constraints (25c)-(25e) are, respectively, associated with scalar variables $Q_{b, i}, P_{i, k^{\prime}}$, and $Q_{i, k^{\prime}}$.

2) Dual OPF: Dual problem of single-objective OPF problem considering the minimization of loss as an objective function

$$
\begin{aligned}
& \varphi\left(Z, R, R^{\prime}\right):=\sum_{i \in \mathcal{N}}\left\{\underline{\lambda}_{i} P_{g, i}^{\min }-\bar{\lambda}_{i} P_{g, i}^{\max }+\lambda_{P_{\mathrm{Loss}}, i} P_{d, i}\right. \\
& +\underline{\gamma}_{i} Q_{g, i}^{\min }-\bar{\gamma}_{i} Q_{g, i}^{\max }+\gamma_{i} Q_{d, i}+\underline{\mu}_{i}\left(v_{\min }\right)^{2}-\bar{\mu}_{i}\left(v_{\max }\right)^{2} \\
& \left.+\underline{\beta}_{i} Q_{b, i}^{\min }-\bar{\beta}_{i} Q_{b, i}^{\max }\right\} \\
& \sigma t_{P_{\mathrm{Loss}},}-\sum_{(i, k) \in \mathcal{L} \& \notin \mathcal{N}_{\mathcal{T}}}\left\{\left(S_{i, k}^{\max }\right)^{2} \ell_{i, k}^{11}+\ell_{i, k}^{22}+\ell_{i, k}^{33}\right\} \\
& -\sum_{\left(i, k^{\prime}\right) \in \mathcal{N}_{\mathcal{T}}}\left\{\left(S_{i, k^{\prime}}^{\max }\right)^{2} \ell_{i, k^{\prime}}^{11}+\ell_{i, k^{\prime}}^{22}+\ell_{i, k^{\prime}}^{33}\right\}
\end{aligned}
$$

and

$$
\begin{aligned}
& \mathbf{A}\left(Z, R, R^{\prime}\right):=\sum_{i \in \mathcal{N}}\left\{\lambda_{P_{\mathrm{Loss}}, i} \mathbf{Y}_{i}+\gamma_{i} \tilde{\mathbf{Y}}_{i}+\mu_{i} M_{i}+\sigma \sum_{i \in \mathcal{N}} \mathbf{Y}_{i}\right\} \\
& +\sum_{(i, k) \in \mathcal{L} \& \notin \mathcal{N} \mathcal{T}}\left\{2 \ell_{i, k}^{12} \mathbf{Y}_{i, k}+2 \ell_{i, k}^{13} \tilde{\mathbf{Y}}_{i, k}\right\} \\
& +\sum_{\left(i, k^{\prime}\right) \in \mathcal{N}_{T}}\left\{\mathbf{M}_{k^{\prime}}\left(\underline{\tau}_{i, k^{\prime}}\left(T_{i, k^{\prime}}^{\min }\right)^{2}-\bar{\tau}_{i, k^{\prime}}\left(T_{i, k^{\prime}}^{\max }\right)^{2}\right)+\tau_{i, k^{\prime}} \mathbf{M}_{i}\right. \\
& +\chi_{i, k^{\prime}}^{\prime}\left(\mathbf{M}^{\prime}{ }_{i, N+k^{\prime}}-\mathbf{M}_{k^{\prime}, N+i}^{\prime}\right)-\chi^{\prime \prime}{ }_{i, k^{\prime}} \mathbf{M}_{i, k^{\prime}}^{\prime} \\
& \left.-\chi^{\prime \prime \prime}{ }_{i, k^{\prime}} \mathbf{M}^{\prime}{ }_{N+i, N+k^{\prime}}\right\}
\end{aligned}
$$

where $\lambda_{P_{\mathrm{Loss}}, i}:=-\underline{\lambda}_{i}+\bar{\lambda}_{i} \quad \forall i \in \mathcal{N}$. Note that in the primal problem, loss minimization function is considered as

$$
\sum_{i \in \mathcal{N}} \operatorname{tr}\left(\mathbf{Y}_{i} \boldsymbol{W}\right) \leq t_{P_{\text {Loss }}}, t_{P_{\text {Loss }}} \geq 0 \quad \forall i \in \mathcal{N}
$$

where $t_{P_{\mathrm{Loss}}}$ is a scalar variable such as $t_{f_{C}}$. Besides, $\sigma$ is used as the Lagrange multipliers related to the constraint (28). Regarding dual multiplier of constraint (28) in the set of $Z$, the duality of the SDP model of OPF problem is considered as

$$
\begin{aligned}
& \max \varphi\left(Z, R, R^{\prime}\right) \\
& \quad \text { subject to }(27),(25 \mathrm{c})-(25 \mathrm{e}) .
\end{aligned}
$$

3) Dual OPF: Dual problem of multiobjective OPF problem considering the cost minimization as the main objective and loss minimization as the secondary objective based on the $\varepsilon$ constraint method.

The dual formulation of the combined two last OPF problems over the $\varepsilon$-constraint method, as stated in (12), can be obtained as (32). Recall that the cost minimization is specified as the main target, and loss minimization of the transmission losses is defined as a second objective, which is taken into account as a new limitation. The dual model of the final relaxed form of SDP problem is presented as follows:

$$
\begin{aligned}
\varphi & \left(Z, R, R^{\prime}\right):=\sum_{i \in \mathcal{N}}\left\{\underline{\lambda}_{i} P_{g, i}^{\min }-\bar{\lambda}_{i} P_{g, i}^{\max }+\lambda_{f_{C}, i} P_{d, i}\right. \\
+ & \underline{\gamma}_{i} Q_{g, i}^{\min }-\bar{\gamma}_{i} Q_{g, i}^{\max }+\gamma_{i} Q_{d, i}+\underline{\mu}_{i}\left(v_{\min }\right)^{2}-\bar{\mu}_{i}\left(v_{\max }\right)^{2} \\
+ & \left.\underline{\beta}_{i} Q_{b, i}^{\min }-\bar{\beta}_{i} Q_{b, i}^{\max }\right\}+\sum_{i \in \mathcal{G}}\left\{c_{i 0}-R_{f_{C}, i}^{22}\right\} \\
& -\sum_{(i, k) \in \mathcal{L} \& \notin \mathcal{N}_{\mathcal{T}}}\left\{\left(S_{i, k}\right)^{2} \ell_{i, k}^{11}+\ell_{i, k}^{22}+\ell_{i, k}^{33}\right\} \\
& -\sum_{\left(i, k^{\prime}\right) \in \mathcal{N}_{\mathcal{T}}}\left\{\left(S_{i, k^{\prime}}^{\max }\right)^{2} \ell_{i, k^{\prime}}^{11}+\ell_{i, k^{\prime}}^{22}+\ell_{i, k^{\prime}}^{33}\right\}-\eta_{\varepsilon_{2, n_{2}}} \varepsilon_{2, n_{2}}
\end{aligned}
$$

and

$$
\begin{aligned}
& \mathbf{A}\left(Z, R, R^{\prime}\right):=\sum_{i \in \mathcal{N}}\left\{\lambda_{f_{C}, i} \mathbf{Y}_{i}+\gamma_{i} \tilde{\mathbf{Y}}_{i}+\mu_{i} M_{i}\right\} \\
& +\eta_{\varepsilon_{2, n_{2}}} \sum_{i \in \mathcal{N}} \mathbf{Y}_{i}+\sum_{(i, k) \in \mathcal{L} \& \notin \mathcal{N} T}\left\{2 \ell_{i, k}^{12} \mathbf{Y}_{i, k}+2 \ell_{i, k}^{13} \tilde{\mathbf{Y}}_{i, k}\right\} \\
& +\sum_{\left(i, k^{\prime}\right) \in \mathcal{N}_{T}}\left\{\mathbf{M}_{k^{\prime}}\left(\underline{\tau}_{i, k^{\prime}}\left(T_{i, k^{\prime}}^{\min }\right)^{2}-\bar{\tau}_{i, k^{\prime}}\left(T_{i, k^{\prime}}^{\max }\right)^{2}\right)+\tau_{i, k^{\prime}} \mathbf{M}_{i}\right. \\
& +\chi_{i, k^{\prime}}^{\prime}\left(\mathbf{M}_{i, N+k^{\prime}}^{\prime}-\mathbf{M}_{k^{\prime}, N+i}^{\prime}\right)-\chi^{\prime \prime}{ }_{i, k^{\prime}} \mathbf{M}_{i, k^{\prime}}^{\prime} \\
& \left.-\chi^{\prime \prime \prime}{ }_{i, k^{\prime}} \mathbf{M}^{\prime}{ }_{N+i, N+k^{\prime}}\right\} .
\end{aligned}
$$

Dual form of SDP problem can be expressed as a linear function to be maximized as

$$
\begin{aligned}
& \max \varphi\left(Z, R, R^{\prime}\right) \\
& \quad \text { subject to }(31),(25 \mathrm{c})-(25 \mathrm{e}) .
\end{aligned}
$$

4) Dual OPF (iv): Dual problem of multiobjective $O P F$ problem considering the cost function minimization as the main objective and loss as well as the pollutant emission minimization as the other objectives based on the $\varepsilon$-constraint method.

Considering a scalar $t_{f_{E}, i} \geq 0$ for every $i \in \mathcal{G}$ to define the convex quadratic emission function as an inequality constraint by the epigraph formulation $f_{E}\left(\mathbf{P}_{\mathbf{g}}\right) \leq \sum_{i \in \mathcal{G}} t_{f_{E}, i}$. Now, this new constraint can be rewritten by Schur's complement formula 
to turn it as an SDP constraint

$$
\begin{aligned}
& {\left[\begin{array}{cc}
e_{i 1} \operatorname{tr}\left(\mathbf{Y}_{i} W\right)-t_{f_{E}, i}+a_{f_{E}, i} & \sqrt{e_{i 2}} \operatorname{tr}\left(\mathbf{Y}_{i} W\right)+b_{f_{E}, i} \\
\sqrt{e_{i 2}} \operatorname{tr}\left(\mathbf{Y}_{i} W\right)+b_{f_{E}, i} & -1
\end{array}\right]} \\
& \preceq 0 \quad \forall i \in \mathcal{G}
\end{aligned}
$$

where

$$
\left\{\begin{array}{l}
a_{f_{E}, i}:=e_{i 0}+e_{i 1} P_{d, i} \\
b_{f_{E}, i}:=\sqrt{e_{i 2}} P_{d, i} .
\end{array}\right.
$$

Moreover, (25d) can be expressed based on the scalar $t_{f_{E}, i}$ as follows:

$$
\sum_{i \in \mathcal{G}} t_{f_{E}, i} \leq \varepsilon_{3, n_{3}}, \quad n_{3}=0,1, \ldots, p_{3} .
$$

The Lagrange multipliers corresponding to the linear matrix inequality (35) are defined as a $2 \times 2$ symmetric matrix

$$
R_{f_{E}, i}:=\left[\begin{array}{cc}
R_{f_{E}, i}^{11} & R_{f_{E}, i}^{12} \\
R_{f_{E}, i}^{12} & R_{f_{E}, i}^{22}
\end{array}\right]
$$

and also, the Lagrange multiplier associated with (34) is denoted by $\vartheta_{\varepsilon_{3, n 3}}$. Then, considering the dual multiplier of (33) in the set of $R$ and the Lagrange multipliers of constraints (21c) and (34) in the set of $Z$, real-valued function $\varphi$ and linear matrix inequality $\mathbf{A}$ can be defined as follows:

$$
\begin{aligned}
\varphi\left(Z, R, R^{\prime}\right):=\sum_{i \in \mathcal{N}}\left\{\underline{\lambda}_{i} P_{g, i}^{\min }-\bar{\lambda}_{i} P_{g, i}^{\max }+\lambda_{f_{C}, i} P_{d, i}+\underline{\gamma}_{i} Q_{g, i}^{\min }\right. \\
-\bar{\gamma}_{i} Q_{g, i}^{\max }+\gamma_{i} Q_{d, i}+\underline{\mu}_{i}\left(v_{\min }\right)^{2}-\bar{\mu}_{i}\left(v_{\max }\right)^{2} \\
\left.+\underline{\beta}_{i} Q_{b, i}^{\min }-\bar{\beta}_{i} Q_{b, i}^{\max }\right\}+\sum_{i \in \mathcal{G}}\left\{c_{i 0}-R_{f_{C}, i}^{22}\right\} \\
-\sum_{(i, k) \in \mathcal{L} \& \notin \mathcal{N}_{\mathcal{T}}}\left\{\left(S_{i, k}\right)^{2} \ell_{i, k}^{11}+\ell_{i, k}^{22}+\ell_{i, k}^{33}\right\} \\
-\sum_{\left(i, k^{\prime}\right) \in \mathcal{N}_{\mathcal{T}}}\left\{\left(S_{i, k^{\prime}}^{\max }\right)^{2} \ell_{i, k^{\prime}}^{11}+\ell_{i, k^{\prime}}^{22}+\ell_{i, k^{\prime}}^{33}\right\} \\
-\eta_{\varepsilon_{2, n_{2}} \varepsilon_{2, n_{2}}+\vartheta_{\varepsilon_{3, n 3}} \varepsilon_{3, n_{3}}}
\end{aligned}
$$

and

$$
\begin{aligned}
& \mathbf{A}\left(Z, R, R^{\prime}\right):=\sum_{i \in \mathcal{N}}\left\{\lambda_{M O, i} \mathbf{Y}_{i}+\gamma_{i} \tilde{\mathbf{Y}}_{i}+\mu_{i} M_{i}\right\} \\
& +\eta_{\varepsilon_{2, n_{2}}} \sum_{i \in \mathcal{N}} \mathbf{Y}_{i}+\sum_{(i, k) \in \mathcal{L} \& \notin \mathcal{N} \mathcal{T}}\left\{2 \ell_{i, k}^{12} \mathbf{Y}_{i, k}+2 \ell_{i, k}^{13} \tilde{\mathbf{Y}}_{i, k}\right\} \\
& +\sum_{\left(i, k^{\prime}\right) \in \mathcal{N}_{T}}\left\{\mathbf{M}_{k^{\prime}}\left(\underline{\tau}_{i, k^{\prime}}\left(T_{i, k^{\prime}}^{\min }\right)^{2}-\bar{\tau}_{i, k^{\prime}}\left(T_{i, k^{\prime}}^{\max }\right)^{2}\right)+\tau_{i, k^{\prime}} \mathbf{M}_{i}\right. \\
& +\chi_{i, k^{\prime}}^{\prime}\left(\mathbf{M}_{i, N+k^{\prime}}^{\prime}-\mathbf{M}_{k^{\prime}, N+i}^{\prime}\right)-\chi_{i, k^{\prime}}^{\prime \prime} \mathbf{M}_{i, k^{\prime}}^{\prime} \\
& \left.-\chi^{\prime \prime \prime}{ }_{i, k^{\prime}} \mathbf{M}^{\prime}{ }_{N+i, N+k^{\prime}}\right\}
\end{aligned}
$$

where

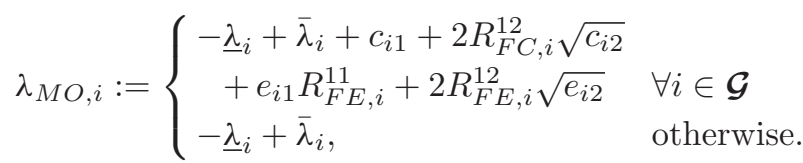

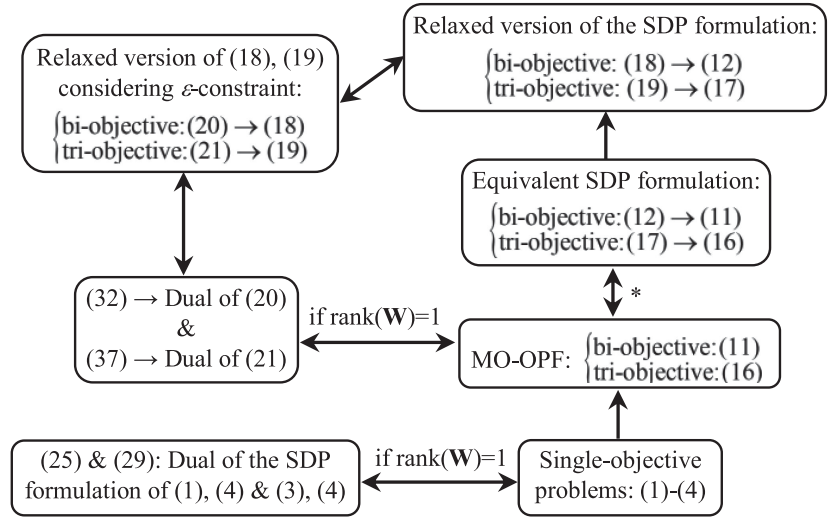

Fig. 1. Connection among different presented OPF optimization problems $(*: \leftrightarrow$ shows the equivalence).

Now, the dual form of the triobjective OPF problem based on $\mathrm{SDP}$ is then given by

$$
\begin{aligned}
& \max \varphi\left(Z, R, R^{\prime}\right) \\
& \quad \text { subject to }(36),(25 c)-(25 e) .
\end{aligned}
$$

Assume that there is no duality gap for every typical OPF problem corresponding to the configuration of the admittance matrix $\mathbf{Y}$. Then, the duality gap is zero for the convex model of MO-OPF problems (32) and (37) as well so that the globally optimal solutions of these problems can be retrieved in the polynomial time. This means that $\operatorname{dim}\left(\operatorname{null}\left(\mathbf{A}\left(Z, R, R^{\prime}\right)\right) \leq 2\right.$ is satisfied in each subproblem produced by the $\varepsilon$-constrained method and then owing to the complementary slackness condition, we have $\operatorname{tr}(\mathbf{A} \boldsymbol{W})=0$ [29], $\operatorname{thusrank}(\boldsymbol{W}) \leq 2$. "null" and "dim" denote, respectively, the null space and the dimensions of the given matrix. At long last, if binding voltage magnitude constraint [30] or other binding constraints [31] are identified, and on the other hand, if reference bus angle is assumed zero, then a unique rank-one solution can be extracted from rank-two solutions in each subproblem of the MO-OPF problem. Fig. 1 demonstrates plainly all of the reformulation steps.

\section{Test Results}

To test the effectiveness of the proposed single and the MO-OPF models, different benchmark problems including the standard IEEE 30-, 57-, and 118-bus test systems and also a real Indian utility 62-bus test system have been considered with the following characteristics.

1) The IEEE 30-bus system comprises 30 buses, 41 branches, nine reactive power compensators, four transformers, and six generators. This optimization problem has 24 variables. The operation data of the IEEE 30-bus system are available in [32].

2) For the IEEE 57-bus system, the limits of the control variables, along with the other necessary data, are extracted from [33]. This system consists of 80 transmission lines, seven generators at buses $1-3,6,8,9$, and 12 , and 15 branches are under load tap setting transformer. Shunt VAR injections are considered at the buses 18, 25, and 53. The total load demand for the system is $1250.8 \mathrm{MW}$ and 336.4 MVAR.

3) IEEE 118-test system is including 54 thermal units, 64 load buses, and 186 transmission lines. The necessary 


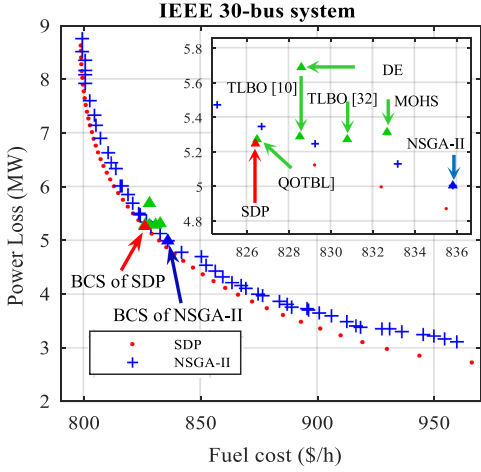

(a)

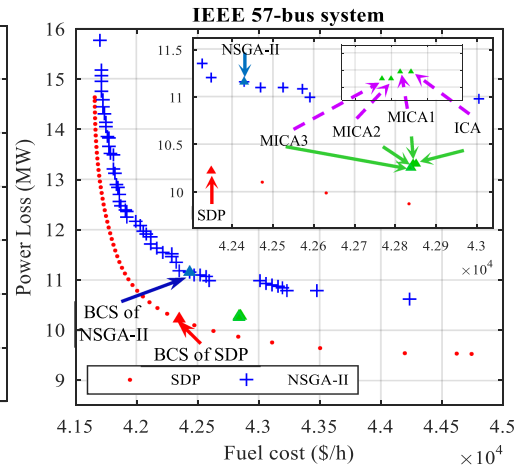

(b)

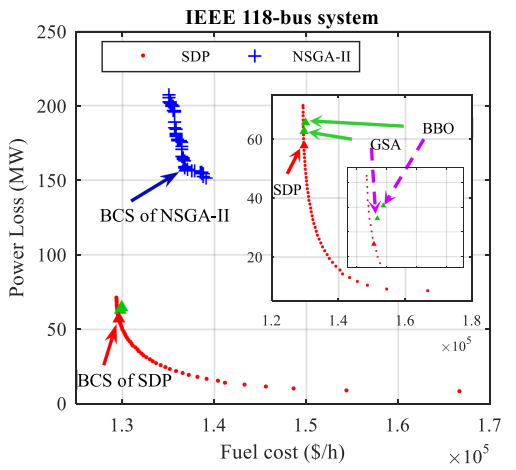

(c)

Fig. 2. Pareto-optimal front of the SDP and NSAGA-II algorithms for (a) IEEE 30-bus test system, (b) IEEE 57-bus test system, and (c) IEEE 118-bus test system.

data of this system are available in [13]. This system consists of 129 control variables, namely 54 active powers of generators and bus voltages, nine taps of tap-changer transformers, and 12 shunt reactive power sources.

4) Indian utility 62-bus system [22], [34] consists of 19 generating units, 89 lines, 11 tap-changing transformers, and nine possible injected VAR sources. The taps of transformers can be taken between 0.9 p.u. and 1.1 p.u. and reactive power sources, each having 30 MVAr capacity that is installed at bus numbers $12,13,15,17,20,21,23$, 24 , and 29.

For a better comparison of the results, the simulations are carried out on two different cases categorized as cases I and II; Case I: In this case study, the MO-OPF model with fuel cost and loss minimization as a biobjective optimization framework is optimized, Case II: In this case, the problem with fuel cost, transmission loss, and emission minimization as three competing objectives is optimized. For all simulations, MATLAB-based toolbox "YALMIP" with the solver "SeDuMi" 1.3 as an SDP solver with tolerance parameter eps $10^{-8}$ is used. In the pursuance of making a better comparison, the nondominated sorting genetic algorithm II (NSGA-II) [35], [36] has been used to solve the MO-OPF problems in the same run environment.

Furthermore, the results of the proposed model are compared with the results of several state-of-the-art algorithms. The parameters of NSGA-II are set as follows.

The maximum generation and the population size are 220 and 50 for both the IEEE 30-bus, 57-bus, and Indian utility 62-bus system; 320 and 520 for the IEEE 118-bus system, respectively

1) crossover distribution index and mutation distribution index are selected 0.8 ;

2) the number of the run for each case study is chosen 50 .

All simulations are performed in MATLAB software (R2016a version) on a laptop with a 64-bit Intel-7500U CPU, Core i7, 2.90-GHz CPU, and 12 GB of RAM.

\section{A. Case I: Biobjective OPF Optimization}

Recall that the first step in the $\varepsilon$-constraint method is to construct the payoff table [25]. To this end, it is essential to obtain the extreme values of both objectives, and initially conduct the SDR of single-objective problems. In accordance with the article presented in [30], $10^{-5}$ p.u. resistance is added to the lines with zero resistance for multiobjective problems. Accordingly, in pursuance of determining the maximum spread of the SDP algorithm, we have added the least resistance to these lines in the single-objective modeling. The ideal minimum points obtained for single-objective problems considering the operating and security constraints for the IEEE 30-, 57-, and 118-bus systems are shown in Fig. 2. To analyze how SDP based on the $\varepsilon$-constrained method behaves with the multiobjective problems, in addition to the cost minimization, which is an integral objective function of the power systems, we are also interested in minimizing the total loss minimization as a competing objective function.

Generally, the experiments aim to evaluate two leading properties of the SDP model: First, the ability to find adequate Pareto fronts for different-scale problems. Second, the required running time for obtaining the nondominated solutions. In order to thoroughly assess the proposed model based on the SDP, three different-scale (IEEE 30-, 57-, and 118-bus) systems have been considered in this article. Optimal compromise solutions of all test cases selected by Fuzzy multicriteria decision makers have been listed in Table I. Notice that having the multiple objectives, a set of outcomes are obtained. So, a tradeoff condition should be achieved between the different outcomes in which the most compromise solution should be determined. This procedure is handled through the fuzzification process. This alternative satisfies all objectives in the best way possible in contrast to the other ones. The total membership function (total degree of optimality) of each Pareto optimal solution is calculated regarding the individual membership functions and the relative importance of the objective functions $\left(w_{i}\right)$. More detailed information about this process can be found in [39]. Table I provides a comparison of the results obtained using the SDP model in the present article with NSGA-II and those reported in the literature for multiobjective cases.

The best compromises of SDP are displayed in boldface. Fig. 2(a)-(c) represents a representative set of Pareto fronts computed by SDP and NSGA-II for all case systems. The horizontal axes denote the cost minimization objective, while the minimization of active loss is displayed on the vertical axes. The BCS presented in Table I have been marked in this figure, and for better clarity, these graphs are redrawn. Analyzing the results mentioned above, the interpretation of associated Pareto front approximations for all case systems leads to three points as follows.

1) For each test case, all solutions that are not all dominated in the Pareto set obtained by SDP satisfy the numerical rank-one condition, and the globally optimal point for each scenario can be acquired in polynomial time. However, 
TABLE I

COMPARISON OF THE BCS FOR IEEE 30-, 57-, AND 118-BUS TEST SySTEMS

\begin{tabular}{|l|l|l|l|l|l|l|l|l|}
\hline IEEE 30-bus system & \multicolumn{4}{l|}{ IEEE 57-bus system } & \multicolumn{3}{l|}{ IEEE 118-bus system } \\
\hline Method & Fuel cost $(\$ / \mathrm{h})$ & Loss (MW) & Method & Fuel cost $(\$ / h)$ & Loss (MW) & Method & Fuel cost (\$/h) & $\begin{array}{l}\text { Loss } \\
(\mathrm{MW})\end{array}$ \\
\hline QOTLBO [10] & 826.4954 & 5.2727 & ICA [33] & 42850.6407 & 10.289 & BBO [13] & 129895 & 65.30 \\
\hline MOHS [37] & 832.6709 & 5.3143 & MICA1 [33] & 42844.7924 & 10.2903 & GSA [13] & 129692 & 62.72 \\
\hline TLBO [10] & 828.5300 & 5.2883 & MICA2 [33] & 42839.7012 & 10.2515 & NSGA-II & 136770.2628 & 157.2435 \\
\hline TLBO [32] & 830.7813 & 5.2742 & MICA3 [33] & 42834.7597 & 10.2511 & SDP & $\mathbf{1 2 9 5 7 6 . 2 8 9 9}$ & $\mathbf{5 7 . 5 2 0 8}$ \\
\hline DE [38] & 828.5900 & 5.6900 & NSGA-II & 43006.0708 & 10.9723 & & & \\
\hline NSGA-II & 826.6931 & 5.3477 & SDP & $\mathbf{4 2 3 5 0 . 5 9 8 8}$ & $\mathbf{1 0 . 2 1 4 8}$ & & & \\
\hline SDP & $\mathbf{8 2 6 . 4 5 9 8}$ & $\mathbf{5 . 2 4 8 4}$ & & & & & & \\
\hline
\end{tabular}

TABLE II

COMPARISON OF PERFORMANCE METRICS

\begin{tabular}{|l|l|l|l|l|l|l|}
\hline Method & \multicolumn{4}{|l|}{ SDP } & \multicolumn{3}{l|}{ NSGA-II } \\
\hline Metric & 30 -bus & 57 -bus & 118-bus & 30-bus & 57-bus & 118-bus \\
\hline$S$ & 0.0024 & 0.0000 & 0.1876 & 0.0056 & 0.0374 & 0.5627 \\
\hline$C$ (SDP,NSGA) & 0.0600 & 0.2000 & 0.8400 & - & - & - \\
\hline$C$ (NSGA,SDP) & - & - & - & 0 & 0 & 0 \\
\hline
\end{tabular}

the SDP model based on the $\varepsilon$-constraint method is able to generate a global Pareto frontier with well distribution, as shown in Fig. 2. A comparison of performance metrics between SDP and NSGA-II shows exactly how uniformly the nondominated fronts are distributed. The spacing [40] metric $(S)$, which determines how uniformly the nondominated front are distributed, and the set coverage [41] metric $[C(M, N)]$, which computes the ratio of Pareto points in $N$ that are weakly dominated by solutions of $M$, validate the even distribution of SDP. These metrics are tabulated in Table II.

2) The SDP method pays off for both cost and loss functions. Since these functions conflict with each other, a significant improvement of one function brings about a substantial degradation in the other one. As an example, for the IEEE 30-bus system, the best compromise alternative can be finally created with either a tradeoff between a $27.5916 \$ / \mathrm{h}$ increase of fuel cost and a $3.2738 \mathrm{MW}$ reduction in loss with regard to the first generation, or a simultaneous loss degradation of $2.4982 \mathrm{MW}$ with $135.4666 \$ / \mathrm{h}$ cutback in fuel cost to the following extreme solution. As another instance, for case 57-bus, SDP obtains the best tradeoff between objectives at a point with $42350.5988 \$ / \mathrm{h}$ and $10.2148 \mathrm{MW}$, i.e., fuel cost is $1.5477 \%$ less than NSGA-II, 1.1432\% less than MICA3 [33], 1.1549\% less than MICA2 [33], 1.1669\% less than MICA1 [33], and $1.1807 \%$ less than ICA [33], and transmission losses is $7.4158 \%$ less than NGSA-II, $0.3554 \%$ less than MICA3 [33], $0.3593 \%$ less than MICA2 [33], $0.7391 \%$ less than MICA1 [33], and $0.7264 \%$ less than ICA [33]. Furthermore, note that the transmission losses achieved by NSGA-II for 118-bus test system (157.8906 MW) in its BCS are even higher than the extreme solution obtained by SDP, in terms of both cost and transmission loss minimization.

3) The Pareto solutions obtained by the SDP model outperforms the nondominated solutions achieved by NSGA-II in all cases and can generate much better sequences. In other words, at the same level of the cost, the transmission
TABLE III

Computed Time for Various Multiobjective Problems in Minutes

\begin{tabular}{|l|l|l|l|}
\hline \multirow{2}{*}{ Method } & \multicolumn{3}{|l|}{ Systems } \\
\cline { 2 - 4 } & 30 -bus & 57 -bus & 118 -bus \\
\hline SDP & 1.11 & 1.75 & 9.81 \\
\hline NSGA-II & 1.31 & 2.29 & 9.63 \\
\hline
\end{tabular}

loss attained by the SDP model is lower than that of the well-known NSGA-II algorithm. At the other end of the scale, the performance of the proposed SDP-based MO-OPF algorithm is compared with those of the other state-of-the-art algorithms, such as QOTLBO [10], MOHS [10], TLBO [10], DE [7], ICA [33], MICA (1,2, and 3) [33], BBO [13], and GSA [13]. From Table I and Fig. 2 can be best seen that the produced solutions of the SDP model easily dominate the best compromising cost and loss of those found by other methods for all test cases 30 , 57 , and 118 .

Especially, the difference is noticeable clearly with increasing the size and complexity of the systems. Notice that the IEEE 118bus system has been demonstrated similar characteristics such as the small and medium case studies, even though this case has higher computational complexity. This shows the accuracy and superiority of the SDP-based method and its ability to generate stronger Pareto optimum.

4) Finally, Table III demonstrates the execution time of various case studies associated with the multiobjective structure in minutes. As observed in this table, although the calculated time for the SDP model is lower or near than NSGA-II, the solver time of the proposed model increases exponentially both in multiobjective and single-objective problems [41].

This shows the main drawback of the standard SDP, especially in cases where problems with larger scales are presented. However, it is worth mentioning that a slight reduction in the accuracy of SeDuMi can lead to a significant reduction in computational time. However, the Pareto set is generally obtained offline; hence, the computational burden is not limitative.

\section{B. Case II: Triobjective OPF Optimization}

The next set of numerical analysis involves applying the triobjective SDP-based formulation presented in dual OPF (iv) on two test systems. For the sake of brevity, a more-detailed comparison based on the most preferred solution between the SDP model and NSGA-II is given in Table IV. Although the Pareto front consists of 50 efficient solutions, only one solution is chosen in order of 
TABLE IV

COMPARISON OF THE BCS FOR IEEE 30 AND INDIAN UTILITY 62-Bus TEST SYSTEMS

\begin{tabular}{|c|c|c|c|c|c|c|c|}
\hline \multirow{2}{*}{ Weight factors } & \multicolumn{4}{|c|}{ IEEE 30-bus system } & \multicolumn{3}{|c|}{ Indian utility 62-bus system } \\
\hline & Method & Fuel cost $(\$ / h)$ & Emission $(\mathrm{lb} / \mathrm{h})$ & Loss (MW) & Fuel cost $(\$ / \mathrm{h})$ & Emission (lb/h) & Loss (MW) \\
\hline \multirow{2}{*}{$w_{\text {Cost }}=w_{\text {Emission }}=w_{\text {Loss }}=0.333$} & NSGA-II & 1416.4241 & 346.7654 & 4.1336 & 15520.4774 & 7333.5409 & 52.9657 \\
\hline & SDP & 864.3504 & 340.7090 & 4.0675 & 13904.9520 & 5851.0903 & 38.9272 \\
\hline \multirow{2}{*}{$w_{\text {Cost }}=0.500, w_{\text {Emission }}=w_{\text {Loss }}=0.250$} & NSGA-II & 838.8434 & 343.5342 & 5.1330 & 14776.0339 & 7131.5816 & 59.2174 \\
\hline & SDP & 814.2541 & 356.9479 & 5.9255 & $\mathbf{1 3 4 6 7 . 1 8 2 5}$ & 6425.4361 & 39.9672 \\
\hline
\end{tabular}

preference. To do so, two sets of weight factors including the same importance for all objective functions as $w_{\text {Cost }}=w_{\text {Emission }}$ $=w_{\text {Loss }}=0.333$ and two times more important for minimization of cost fuel in comparison to the other objective as $w_{\text {Cost }}=$ $0.500, w_{\text {Emission }}=w_{\text {Loss }}=0.250$ are considered in Table IV. As expected, due to a change of importance of objective functions, the production cost is reduced from $864.3504(\$ / \mathrm{h})$ and $13904.9520(\$ / \mathrm{h})$ to $814.2541(\$ / \mathrm{h})$ and $13467.1825(\$ / \mathrm{h})$ (when the objective functions have the same importance) for the IEEE 30-bus and the Indian utility 62-bus test systems, respectively. Looking at this table, some thought-provoking remarks can be inferred: First, with both sets of weight factors, the BCS obtained by SDP outperforms the NSGA-II technique. Second, it was found in the numerical analysis that adding more objective functions does not degrade the performance of the proposed model, and the results corroborate that the SDP-based model is also a dependable tool to solve the real-world multiobjective OPF problems, where multiple Pareto front solutions can be achieved by changing $\varepsilon$.

\section{CONCLUSION}

This article has provided a contribution to extend the convex multiobjective models for the OPF problems based on SDP. The $\varepsilon$-constrained method is effectively implemented for approximating the Pareto front solutions. Actually, this article tries to prove that a zero duality gap for more complex power problems can also be kept. Considered that the nonlinear objective functions of the MO-OPF problem are the total fuel cost, transmission losses, and emission pollutants, where the minimization of fuel cost is regarded as the leading objective, and the other objectives are added to other inequality constraints of the OPF problem. This article addresses the single-objective problems at first with modeling all security and operational constraints, more especially, considering control parameters, such as tap-changing transformers and variable shunt elements. After obtaining the extreme solutions, the nonconvex multiobjective OPF problem based on the $\varepsilon$-constrained method is modeled in a convex way using SDP. It has shown that the proposed modeling can be successfully applied to various scale systems, and all nondominated solutions in the Pareto set satisfy the rank condition for each scenario produced by the $\varepsilon$-constrained method and the obtained BCS are better than those of the other methods. Moreover, comparison metrics to assess the diversity property of Pareto front obtained by SDP, NSGA-II, and other widely known algorithms verify the computational efficacy, accuracy, and applicability of the SDP-based framework.

\section{APPENDIX}

As a reminder, $\mathbf{Y}_{i}, \tilde{\mathbf{Y}}_{i}, \mathbf{Y}_{i k}, \tilde{\mathbf{Y}}_{i k}$, and $\mathbf{M}_{i}$, which are used in the processing of reformulation the OPF equations based on
SDP, are defined as follows according to [24]:

$$
\begin{aligned}
\mathbf{Y}_{i}:=\frac{1}{2}\left[\begin{array}{ll}
\operatorname{Re}\left\{Y_{i}+Y_{i}^{T}\right\} & \operatorname{Im}\left\{Y_{i}^{T}-Y_{i}\right\} \\
\operatorname{Im}\left\{Y_{i}-Y_{i}^{T}\right\} & \operatorname{Re}\left\{Y_{i}+Y_{i}^{T}\right\}
\end{array}\right] \\
\tilde{\mathbf{Y}}_{i}:=\frac{-1}{2}\left[\begin{array}{ll}
\operatorname{Im}\left\{Y_{i}+Y_{i}^{T}\right\} & \operatorname{Re}\left\{Y_{i}-Y_{i}^{T}\right\} \\
\operatorname{Re}\left\{Y_{i}^{T}-Y_{i}\right\} & \operatorname{Im}\left\{Y_{i}+Y_{i}^{T}\right\}
\end{array}\right] \\
\mathbf{Y}_{i, k}:=\frac{1}{2}\left[\begin{array}{ll}
\operatorname{Re}\left\{Y_{i, k}+Y_{i, k}^{T}\right\} & \operatorname{Im}\left\{Y_{i, k}^{T}-Y_{i, k}\right\} \\
\operatorname{Im}\left\{Y_{i, k}-Y_{i, k}^{T}\right\} & \operatorname{Re}\left\{Y_{i, k}+Y_{i, k}^{T}\right\}
\end{array}\right] \\
\tilde{\mathbf{Y}}_{i, k}:=\frac{-1}{2}\left[\begin{array}{cc}
\operatorname{Im}\left\{Y_{i, k}+Y_{i, k}^{T}\right\} & \operatorname{Re}\left\{Y_{i, k}-Y_{i, k}^{T}\right\} \\
\operatorname{Re}\left\{Y_{i, k}^{T}-Y_{i, k}\right\} & \operatorname{Im}\left\{Y_{i, k}+Y_{i, k}^{T}\right\}
\end{array}\right] \\
M_{i}:=\left[\begin{array}{cc}
e_{i} e_{i}^{T} \quad 0 \\
0 & \left.e_{i} e_{i}^{T}\right] .
\end{array}\right.
\end{aligned}
$$

If $P_{d, i}+j Q_{d, i}$ is considered as the connected load to the bus $i$, and also the injected power to bus $i$ are regarded as $P_{\text {inj }, i}=P_{g, i}-P_{d, i}$ and $Q_{\text {inj, } i}=Q_{g, i}-Q_{d, i}$, then the following equations can be obtained $\forall i \in \mathcal{N}$ and $\forall(i, k) \in \mathcal{L}$ :

$$
\begin{aligned}
P_{\mathrm{inj}, i} & =\operatorname{tr}\left(\mathbf{V}^{T} \mathbf{Y}_{i} \mathbf{V}\right) \\
Q_{\mathrm{inj}, i} & =\operatorname{tr}\left(\mathbf{V}^{T} \tilde{\mathbf{Y}}_{i} \mathbf{V}\right) \\
P_{i, k} & =\operatorname{tr}\left(\mathbf{V}^{T} \mathbf{Y}_{i, k} \mathbf{V}\right) \\
Q_{i, k} & =\operatorname{tr}\left(\mathbf{V}^{T} \tilde{\mathbf{Y}}_{i, k} \mathbf{V}\right) \\
\left|S_{i, k}\right|^{2} & =\left\{\operatorname{tr}\left(\mathbf{V}^{T} \mathbf{Y}_{i, k} \mathbf{V}\right)\right\}^{2}+\left\{\operatorname{tr}\left(\mathbf{V}^{T} \tilde{\mathbf{Y}}_{i, k} \mathbf{V}\right)\right\}^{2} \\
\left|v_{i}\right|^{2} & =\operatorname{tr}\left(\mathbf{V}^{T} \mathbf{M}_{i} \mathbf{V}\right) .
\end{aligned}
$$

Now, using these matrices and by defining $W:=\mathbf{V V}^{T}$, problem (4) can be easily reformulated as problem (12) based on SDP.

\section{REFERENCES}

[1] H. R. Esmaeilian and R. Fadaeinedjad, "Energy loss minimization in distribution systems utilizing an enhanced reconfiguration method integrating distributed generation," IEEE Syst. J., vol. 9, no. 4, pp. 1430-1439, Dec. 2015.

[2] A. M. Shaheen, R. A. El-Sehiemy, and S. M. Farrag, "Solving multiobjective optimal power flow problem via forced initialised differential evolution algorithm," IET Gener., Transmiss. Distrib., vol. 10, no. 7, pp. 1634-1647, May 2016.

[3] A. M. L. da Silva and J. G. de Carvalho Costa, "Transmission loss allocation: I-Single energy market," IEEE Trans. Power Syst., vol. 18, no. 4, pp. 1389-1394, Nov. 2003. 
[4] S. S. Reddy, "Multi-objective based congestion management using generation rescheduling and load shedding," IEEE Trans. Power Syst., vol. 32 no. 2, pp. 852-863, Mar. 2017.

[5] A. Yazdaninejadi, A. Hamidi, S. Golshannavaz, F. Aminifar, and S. Teimourzadeh, "Impact of inverter-based DERs integration on protection, control, operation, and planning of electrical distribution grids," Elect. J., vol. 32, pp. 43-56, 2019.

[6] A. Hamidi, D. Nazarpour, and S. Golshannavaz, "Multiobjective scheduling of microgrids to harvest higher photovoltaic energy," IEEE Trans. Ind. Inform., vol. 14, no. 1, pp. 47-57, Jan. 2017.

[7] R. Moslemi, M. Esmaili, H. A. Shayanfar, L. Wang, and R. C. Green, "Multi-objective environmental optimal power flow considering transient stability improvement," Int. Rev. Elect. Eng., vol. 7, pp. 3443-3453, 2012.

[8] C. Roman and W. Rosehart, "Evenly distributed Pareto points in multiobjective optimal power flow," IEEE Trans. Power Syst., vol. 21, no. 2, pp. 1011-1012, May 2006.

[9] S. S. Reddy and P. R. Bijwe, "Multi-objective optimal power flow using efficient evolutionary algorithm," Int. J. Emerg. Electr. Power Syst., vol. 18, 2017.

[10] B. Mandal and P. K. Roy, "Multi-objective optimal power flow using quasioppositional teaching learning based optimization," Appl. Soft Comput., vol. 21, pp. 590-606, 2014.

[11] K. Teeparthi and D. M. V. Kumar, "Multi-objective hybrid PSO-APO algorithm based security constrained optimal power flow with wind and thermal generators," Eng. Sci. Technol., Int. J., vol. 20, pp. 411-426, 2017.

[12] L. Shi, C. Wang, L. Yao, Y. Ni, and M. Bazargan, "Optimal power flow solution incorporating wind power," IEEE Syst. J., vol. 6, no. 2, pp. 233241, Jun. 2011.

[13] A. Bhattacharya and P. Roy, "Solution of multi-objective optimal power flow using gravitational search algorithm," IET Gener., Transmiss. Distrib., vol. 6, no. 8, pp. 751-763, Aug. 2012.

[14] S. S. Reddy, P. R. Bijwe, and A. R. Abhyankar, "Multi-objective market clearing of electrical energy, spinning reserves and emission for windthermal power system," Int. J. Elect. Power Energy Syst., vol. 53, pp. 782794, 2013.

[15] S. S. Reddy and P. R. Bijwe, "Differential evolution-based efficient multiobjective optimal power flow," Neural Comput. Appl., vol. 31, pp. 509$522,2019$.

[16] S. Boyd and L. Vandenberghe, Convex Optimization. Cambridge, U.K.: Cambridge Univ. Press, 2004

[17] L. Gan and S. H. Low, "Optimal power flow in direct current networks," IEEE Trans. Power Syst. vol. 29, no. 6, pp. 2892-2904, Nov. 2014.

[18] M. S. Andersen, A. Hansson, and L. Vandenberghe, "Reduced-complexity semidefinite relaxations of optimal power flow problems," IEEE Trans. Power Syst., vol. 29, no. 4, pp. 1855-1863, Jul. 2014.

[19] B. Kocuk, S. S. Dey, and X. A. Sun, "Inexactness of SDP relaxation and valid inequalities for optimal power flow," IEEE Trans. Power Syst., vol. 31, no. 1, pp. 642-651, Jan. 2016.

[20] S. S. Reddy, "Multi-objective based adaptive immune algorithm for solving the economic and environmental dispatch problem," Int. J. Appl. Eng. Res., vol. 12, pp. 1043-1048, 2017.

[21] S. R. Salkuti and S.-C. Kim, "Congestion management using multiobjective glowworm swarm optimization algorithm," J. Elect. Eng. Technol., vol. 14, pp. 1565-1575, 2019.

[22] R. Gnanadass, N. P. Padhy, and K. Manivannan, "Assessment of available transfer capability for practical power systems with combined economic emission dispatch," Electr. Power Syst. Res., vol. 69, pp. 267-276, 2004.

[23] H. Wolkowicz, R. Saigal, and L. Vandenberghe, Handbook of Semidefinite Programming: Theory, Algorithms, and Applications, vol. 27. New York, NY, USA: Springer, 2012.
[24] D. Wu, A. Hu, J. Zhou, and S. Wu, "A new convex relaxation for quadratically constrained quadratic programming," Filomat, vol. 27, pp. 1511$1521,2013$.

[25] C.-L. Hwang and A. S. M. Masud, Multiple Objective Decision MakingMethods and Applications: A State-of-the-Art Survey, vol. 164. New York, NY, USA: Springer, 2012.

[26] G. Mavrotas, "Effective implementation of the $\varepsilon$-constraint method in multi-objective mathematical programming problems," Appl. Math. Comput., vol. 213, pp. 455-465, 2009.

[27] B. Bentouati, S. Chettih, R. E. Sehiemy, and G.-G. Wang, "Elephant herding optimization for solving non-convex optimal power flow problem," $J$. Electr. Electron. Eng., vol. 10, pp. 31-40, 2017.

[28] E. Davoodi, E. Babaei, B. Mohammadi-Ivatloo, and M. Rasouli, “A novel fast semidefinite programming-based approach for optimal reactive power dispatch," IEEE Trans. Ind. Inform., vol. 16, no. 1, pp. 288-298, Jan. 2020.

[29] D. K. Molzahn, B. C. Lesieutre, and C. L. DeMarco, "Investigation of non-zero duality gap solutions to a semidefinite relaxation of the optimal power flow problem," in Proc. 47th Hawaii Int. Conf. Syst. Sci., 2014, pp. 2325-2334.

[30] J. Lavaei and S. H. Low, "Zero duality gap in optimal power flow problem," IEEE Trans. Power Syst., vol. 27, no. 1, pp. 92-107, Feb. 2012.

[31] D. K. Molzahn, J. T. Holzer, B. C. Lesieutre, and C. L. DeMarco, "Implementation of a large-scale optimal power flow solver based on semidefinite programming," IEEE Trans. Power Syst., vol. 28, no. 4, pp. 3987-3998, Nov. 2013.

[32] M. R. Nayak, C. K. Nayak, and P. K Rout, "Application of multiobjective teaching learning based optimization algorithm to optimal power flow problem," Procedia Technol., vol. 6, pp. 255-264, 2012.

[33] M. Ghasemi, S. Ghavidel, M. M. Ghanbarian, H. R. Massrur, and M. Gharibzadeh, "Application of imperialist competitive algorithm with its modified techniques for multi-objective optimal power flow problem: A comparative study," Inf. Sci., vol. 281, pp. 225-247, 2014.

[34] Electric power systems analysis \& nature-inspired optimization algorithm, 2015. [Online]. Available: http://al-roomi.org/power-flow/62-bus-system

[35] K. Deb, A. Pratap, S. Agarwal, and T. Meyarivan, "A fast and elitist multiobjective genetic algorithm: NSGA-II," IEEE Trans. Evol. Comput., vol. 6, no. 2, pp. 182-197, Apr. 2002.

[36] Y.-P. Fang, N. Pedroni, and E. Zio, "Comparing network-centric and power flow models for the optimal allocation of link capacities in a cascade-resilient power transmission network," IEEE Syst. J., vol. 11, no. 3, pp. 1632-1643, Sep. 2014.

[37] S. Sivasubramani and K. S. Swarup, "Multi-objective harmony search algorithm for optimal power flow problem," Int. J. Elect. Power Energy Syst., vol. 33, pp. 745-752, 2011.

[38] M. Abido and N. A. Al-Ali, "Multi-objective optimal power flow using differential evolution," Arabian J. Sci. Eng., vol. 37, pp. 991-1005, 2012.

[39] J. Aghaei, N. Amjady, and H. A. Shayanfar, "Multi-objective electricity market clearing considering dynamic security by lexicographic optimization and augmented epsilon constraint method," Appl. Soft Comput., vol. 11, pp. 3846-3858, 2011.

[40] J. R. Scott, "Fault tolerant design using single and multi-criteria genetic algorithms," M.S. thesis, Dept. Aeronaut. Astronaut., Massachusetts Inst. Technol., Cambridge, MA, USA, 1995.

[41] A. M. Jubril, O. A. Komolafe, and K. O. Alawode, "Solving multi-objective economic dispatch problem via semidefinite programming," IEEE Trans. Power Syst., vol. 28, no. 3, pp. 2056-2064, Aug. 2013. 